\title{
UTENSILIOS Y ESTRUCTURAS RELACIONADOS CON EL CULTIVO Y TRANSFORMACIÓN DE CEREAL EN LA PROTOHISTORIA DEL SUR DE LA PENÍNSULA IBÉRICA
}

\author{
TOOLS AND STRUCTURES RELATED TO CROP AND PROCESSING OF CEREAL \\ IN THE PROTOHISTORY AT SOUTH OF IBERIAN PENINSULA
}

\author{
ANDRÉS ROLDÁN DÍAZ* / ANDRÉS MARÍA ADROHER AUROUX**
}

\begin{abstract}
Resumen: En este trabajo recopilamos los restos de herramientas y estructuras documentadas en la mitad meridional de la península ibérica durante la Protohistoria, relacionados con las distintas fases del proceso de producción del cereal y las inferencias que podemos obtener acerca de la variedad de aspectos relacionados con la preparación, producción, transformación y consumo, en cualquier parcela de la vida de los grupos culturales prerromanos en esta zona. De esta forma nos acercamos a una parte de la cadena operativa, la que puede precisamente reconstruirse a partir de estas evidencias arqueológicas. Los datos procedentes de la arqueobotánica han sido dejados de lado en este estudio, a pesar de que podrían proporcionar una información interesante, pero estimamos que hay demasiadas pocas analíticas convenientemente publicadas en el ámbito espacial y cronológico en que nos movemos, como para establecer generalidades.

Palabras clave: Protohistoria, ibérico, agricultura, cereal, cadena operativa.
\end{abstract}

\section{INTRODUCCIÓN}

El estudio de las culturas prerromanas del sur de la península ibérica varía notablemente dependiendo del espacio geográfico y cronológico en que nos movamos.

\footnotetext{
* CIHAI. Correo-e: andresroldandiaz@gmail.com
}

\begin{abstract}
In this work we collect the remains of tools and structures that have been documented for the southern half of the Iberian Peninsula during the protohistoric period, related to the various phases of processing for grain production. We also compile the inferences which we have been able to draw, regarding a variety of features related to the preparation, production, transformation, and consumption, in each aspect of the lives of the pre-Roman cultural groups in this region. In this way, we get closer to understanding the chaine opératoire, which can be precisely reconstructed using these archaeological data. Information arising from archaeobotanical research has been left out of this study, although they could provide some useful information, because we believe that there have so far been too few well-published analyses for this spatial and chronological context, to allow us to draw general conclusions.
\end{abstract}

Keywords: Protohistory, Iberian, agriculture, cereal, chaîne opératoire.

Mientras en unas regiones, especialmente las del Suroeste, se ha estudiado con mayor detalle el periodo comprendido entre el Bronce Final y el comienzo de la Edad del Hierro, en la zona oriental se conoce mejor la cultura ibérica en su momento de mayor plenitud,

\footnotetext{
** Universidad de Granada. Correo-e: aadroher@ugr.es
} 
siendo las provincias de Jaén y Murcia referentes en el estudio de dicho periodo.

También varía la temática de los trabajos realizados, lo cual ha provocado cierta descompensación en el conocimiento de algunos temas, algunos de los cuales están siendo relegados a un triste segundo plano en la investigación, siendo uno de los aspectos que más en serio nos deberíamos tomar, la casi total ausencia de publicaciones relacionadas con el estudio de diversos temas referidos a los ámbitos domésticos; la mayor parte de las intervenciones arqueológicas que han impactado en la historiografía del último medio siglo de la Protohistoria del Sur peninsular se centran en los hallazgos de los grandes palacios (Montemayor, Cancho Roano o, más recientemente, Puente Tablas), santuarios con interesantes repertorios de materiales muebles (Carambolo, El Cigarralejo o las cuevas de Despeñaperros), necrópolis con ajuares muy atractivos (Baza, Coimbra de Barranco Ancho, El Cigarralejo) o fortificaciones no siempre bien documentadas (Tejada la Vieja, Puente Tablas o Iliberri), pero que se han convertido en los hitos actualmente definitorios de la cultura material (e inmaterial) de las sociedades prerromanas, visión que, a todas luces, presenta un importante sesgo puesto que, una vez más, dentro de la más pura historiografía historicista, parece ponerse más énfasis en el análisis de las altas capas sociales cuyo registro arqueológico es más visible, dando la razón a la poco afortunada denominación de la famosa exposición de 1998, "Iberos, Príncipes de Occidente".

Ello ha provocado la sobrevaloración de los espacios particulares frente a los espacios cotidianos, lo particular sobre lo general, y, por tanto, existe cierta tendencia a conceptualizar cualquier espacio como edificio singular o hecho singular; el problema puede haber llegado a sesgar notablemente los resultados de las investigaciones sobre la estructura y red urbana de los hábitats ibéricos, así como la percepción que poco a poco va impregnando a nuestra sociedad actual respecto a la idea de qué caracterizaría a las sociedades ibéricas.

En este sentido, es probable que algunos espacios identificados como estancias sagradas a partir del valor cualitativo (o cuantitativo) de los materiales localizados durante el proceso de excavación, en realidad debieran ser considerados espacios domésticos en los cuales se da un nivel de conservación del registro arqueológico más completo que en estancias contiguas. Desde ese punto de vista hay que reconocer que el argumento para definir en La Quéjola la existencia de un posible espacio sagrado podría responder a este tipo de conservación diferencial del registro en unidades domésticas
(Blánquez 1995; Moneo 2003: 111-113) más que a una especifidad funcional de dicho ámbito estructural.

De esta forma, algunos estudios sobre religiosidad pecan de un excesivo funcionalismo, estableciendo unos criterios poco elaborados para definir la calidad de sagrado aplicada a ciertos espacios basándose exclusivamente en argumentos de calidad y/o cantidad más que a argumentos contextuales y/o analógicos (Moneo 2003).

En consecuencia de todo lo anterior estamos perdiendo informaciones interesantes acerca del ámbito doméstico, de la producción dentro de la unidad familiar, o para ser más exactos, de las denominadas "actividades de mantenimiento", entendidas como "el conjunto de actividades relacionadas con el sostenimiento y bienestar de los miembros de un grupo social, de tal manera que (...) incluyen todas las actividades cotidianas tales como la recolección, preparación, distribución, consumo y almacenamiento de los alimentos,..." (Falcó Martí 2003: 218, con abundante bibliografía sobre el concepto).

De esta situación, generalizable a otros períodos históricos, se hizo eco Sandra Montón en un crítico artículo acerca de los motivos que han provocado el desplazamiento en la investigación de los ámbitos domésticos en arqueología, incluyendo una fuerte diatriba feminista a las bases que provocaron este abandono (Montón Subías 2005: 159-160); no obstante, existen otros elementos que han afectado igualmente a este desinterés, aunque no es éste el lugar para analizarlos. En este sentido, de un lado el propio registro arqueológico que conserva mejor elementos monumentales o ámbitos funerarios, y por otro, innegable y ciertamente peligroso, las políticas oficiales de investigación, para lo que puede consultarse los escritos del sociólogo francés B. Latour (1991) (con pésima traducción al castellano en 2007).

Por tanto, entendemos que cualquier aportación al conocimiento de los ámbitos domésticos, así como de las actividades relacionadas con lo que actualmente definiríamos como sector económico primario o de producción básica (recolección, caza, pesca, agricultura, ganadería y gestión del agua), significa un importante acercamiento a aspectos que, como decimos, suelen quedar relegados a un segundo plano en las investigaciones más recientes.

\section{METODOLOGÍA, MÉTODO, TÉCNICAS Y OBJETIVOS}

Entendemos como planteamiento de partida que el análisis de los ámbitos domésticos refleja, como pocos, los cambios que se producen inherentes a cualquier 


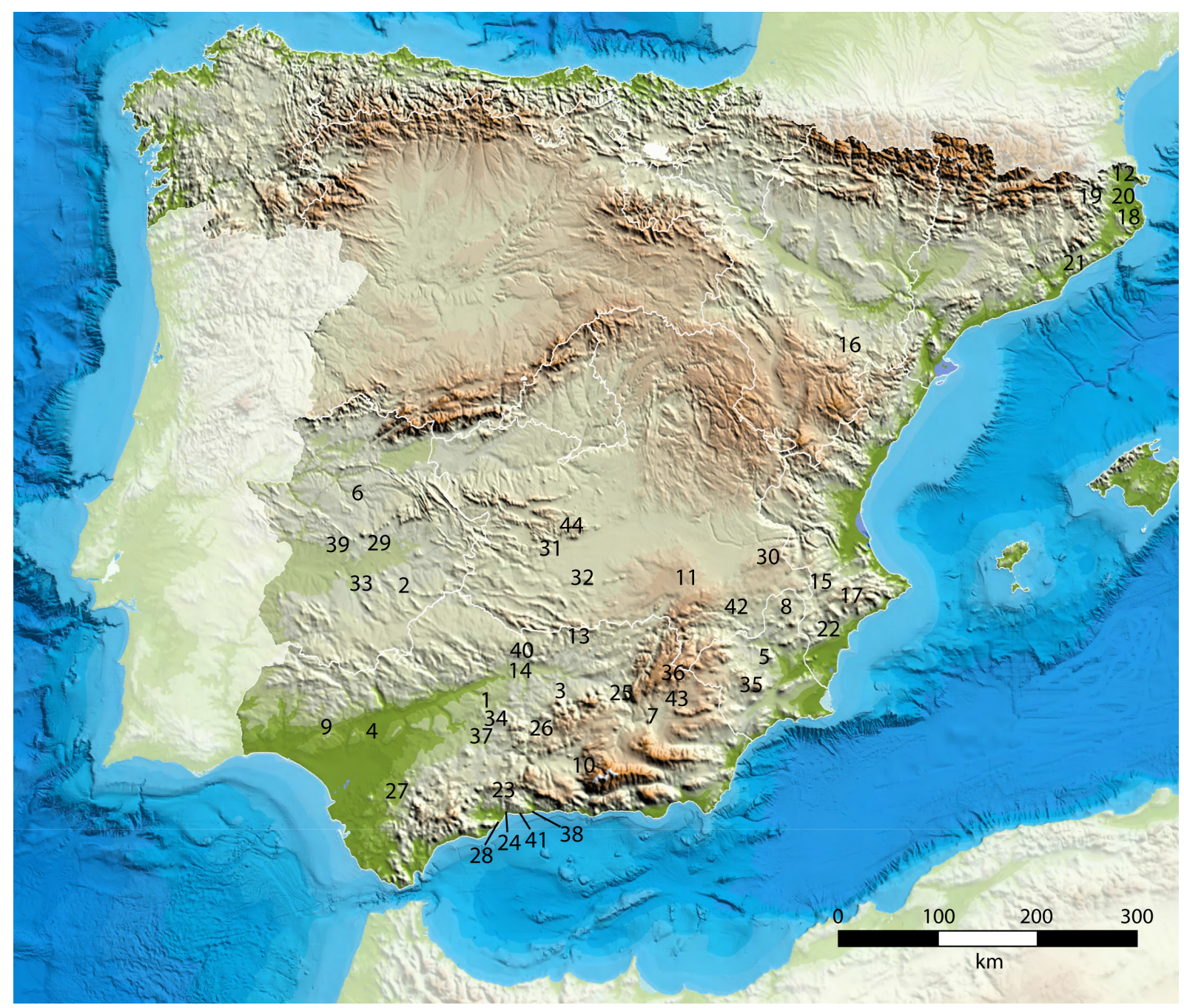

Figura 1. Mapa con los yacimientos mencionados en el texto. 1. Montemayor (Córdoba); 2. Cancho Roano (Zalamea de la Serena, Badajoz); 3. Plaza de Armas de Puente Tablas (Jaén); 4. El Carambolo (Camas, Sevilla); 5. El Cigarralejo (Mula, Murcia); 6. Cáceres el Viejo; 7. Baza (Granada); 8. Coimbra de Barranco Ancho (Jumilla, Murcia); 9. Tejada la Vieja (Escacena del Campo, Huelva); 10. Iliberri (Granada); 11. La Quéjola (San Pedro, Albacete); 12. Mas Castellar de Pontós (Gerona); 13. Collado de los Jardines (Santa Elena, Jaén); 14. Obulco (Porcuna, Jaén); 15. La Bastida de les Alcusses (Mogente, Valencia); 16. Cabezo de la Guardia (Alcorisa, Teruel); 17. Covalta (Albaida, Valencia); 18. Ullastret; 19. Porqueres (Banyoles, Gerona); 20. Ampurias (Gerona); 21. Burriac (Cabrera de Mataró, Barcelona); 22. Peña Negra (Crevillente, Alicante); 23. Cerro de la Capellanía (Periana, Málaga); 24. Cerca Niebla (Vélez-Málaga, Málaga); 25. Castellones de Céal (Hinójares, Jaén); 26. Cerro de la Cruz (Almedinilla, Córdoba); 27. Villamartín (Cádiz); 28. Loma de Benagalbón (Rincón de la Victoria, Málaga); 29. Cerro Manzanillo (Villar de Rena, Badajoz); 30. El Amarejo (Bonete, Albacete); 31. Alarcos (Ciudad Real); 32. Cerro de las Cabezas (Valdepeñas, Ciudad Real); 33. El Palomar (Oliva de Mérida, Badajoz); 34. Plaza de Armas (Nueva Carteya, Córdoba); 35. Murtal de Alhama (Alhama de Murcia, Murcia); 36. Cerro de la Cruz (Bugéjar, Puebla de Don Fadrique, Granada); 37. Monturque (Córdoba); 38. Las Chorreras (Vélez-Málaga, Málaga); 39. El Chaparral (Aljucén, Badajoz); 40. Calañas (Marmolejo, Jaén); 41. Morro de Mezquitilla (Algarrobo, Málaga); 42. Almadenes (Hellín, Albacete); 43. Fuente Amarga (Galera, Granada); 44. Calatrava la Vieja (Carrión de Calatrava, Ciudad Real).

grupo social, independientemente de su complejidad tecnológica, de su contexto social, o del entorno.

Y dentro de las actividades domésticas, los procesos relacionados con la alimentación deben ser objetivos preferentes en la investigación, pues suponen la base de la subsistencia de dichos grupos. Esto motiva que nos queramos acercar al ámbito de la producción de comestibles y, dentro de él, de uno de los que supone un 
importante porcentaje de los nutrientes y aportes de calorías para el mantenimiento de la vida cotidiana: el cereal en su amplio espectro.

Dicho esto, pasamos a definir una serie de conceptos que serán utilizados a lo largo del texto, tomando como base los de la Arqueología de la Producción (Mannoni y Giannicheda 2007), de forma que agilicemos el proceso descriptivo e interpretativo de nuestro discurso, si bien nos permitiremos alguna reformulación de dichos conceptos si comprobamos que en el Diccionario de la Real Academia de la Lengua Española, pudieran existir términos o definiciones más apropiadas para los diversos aspectos que hemos querido describir en este texto.

a) Recurso: materia presente de forma natural en el paisaje y susceptible de ser utilizada tecnológicamente por un grupo cultural, pero que no ha sido alterada química ni físicamente por el ser humano.

b) Materia prima: elemento extraído de la naturaleza y que se transformará para elaborar cualquier tipo de producto consumible por el ser humano. Se trata del primer nivel de alteración del recurso a explotar, pudiéndose dar tanto alteraciones simplemente físicas (cantería) como químicas (metalurgia), pero que no implican en ningún caso un producto de consumo final.

c) Producto: es un objeto u elemento consumible, que se ofrece a un usuario con la intención de satisfacer una necesidad o un deseo, independientemente de que el consumo sea directo (consumo cultural directo) o a través de otros elementos, personas o animales (consumo cultural derivado). Es el último paso de la cadena operativa, aunque también puede retroalimentarla fabricando productos que se conviertan a su vez en materia prima.

d) Subproducto: elemento consecuencia del proceso, como logro secundario o a veces inesperado del procedimiento técnico de fabricación. Puede convertirse a su vez en materia prima o en producto de consumo.

e) Desecho: elemento no consumible y/o irrecuperable técnicamente, por tanto se trata de un residual resultante en cualquier momento de la cadena operativa.

Intentaremos aportar la mayor información posible de la cadena operativa que afecta a la producción, transformación y consumo del cereal en las sociedades prerromanas, tomando datos de los elementos artefactuales localizados en los diversos yacimientos en el Mediodía peninsular.

Por otra parte, entendemos que para el análisis del papel de las gramíneas hay dos ámbitos que deben ser tratados lógicamente: en primer lugar los elementos artefactuales y en segundo lugar los ecofactuales, frecuentemente provenientes de análisis relacionados con la arqueobotánica, y que proporcionan interesantes inferencias de carácter paleoambiental y por ende paleoeconómico, entendiendo, como consideramos desde nuestra perspectiva de la Arqueología del Paisaje, que economía y medio son dos ámbitos culturales indisociables, aunque según otros planteamientos teóricos, podrían evaluarse como categorías de análisis netamente diferenciables, lo que proporcionaría, desde nuestra perspectiva, una visión sesgada de la relación entre una cultura y su entorno.

Igualmente se haría necesario un estudio de arqueozoología, habida cuenta de la relevante información que puede aportar a ciertos procesos donde el tiro tiene una importancia fundamental, como el caso de bueyes, mulos y caballos, tanto en la preparación del terreno de cultivo, la plantación, el mantenimiento, transporte e incluso en otros puntos de la cadena operativa como el papel que juegan en los molinos de sangre; la caza de suidos y lagomorfos para la defensa de los cultivos (al margen de que fueran o no consumido con posterioridad), o las amenazas que representan los roedores en relación a los problemas de almacenaje. No obstante, de la misma forma que sucede en el caso de la arqueobotánica, tampoco las disciplinas relacionadas con los estudios faunísticos (microfauna, macrofauna, ictiofauna o malacología) han trabajado mucho sobre la Protohistoria del Mediodía peninsular.

A pesar de la multiplicidad de datos que los análisis paleobotánicos pueden aportar a este proceso, debemos reconocer que son muy escasos los resultados publicados que proporcionan información respecto al espacio y el tiempo que ocupan a este trabajo. Salvo muy contados casos (Iliberri en Granada, El Turruñuelo y Puente Tablas en Jaén, Cerro del Villar en Málaga, Castillo de Doña Blanca en Cádiz y poco más), casi nada tenemos acerca de la Edad del Hierro, lo que contrasta con la cantidad y calidad de las analíticas practicadas para otros períodos precedentes, especialmente de la Edad del Cobre y Edad del Bronce, descompensación de la que se hacen eco numerosos trabajos (Rovira i Buendía 2007) y que justifica que no utilicemos ese tipo de información, pues esos escasos datos necesitan de una revisión en conjunto y un análisis más detallado de manos de un especialista en arqueobotánica, quien pueda darle el valor y la estimación que le corresponde dentro de los diversos momentos del uso de los vegetales en general y de las gramíneas en particular, en las sociedades prerromanas peninsulares.

Cuando se generalicen las analíticas arqueobotánicas en contextos del primer milenio a.C., obtendremos 
mucha más información acerca de aspectos que no suelen dejar restos directos en el registro arqueológico, llegándose a ellos a través de canales interpretativos indirectos. Así entendemos que informan mejor que las herramientas sobre ciertos procesos de gestión de cultivos, temporadas de siembra y siega, mantenimiento de cultivos, transformación y segregación del grano, transporte y un largo etcétera.

Pero, por el momento, siendo conscientes del sesgo que ello supondría de contar con más datos, hemos decidido limitarnos a la documentación de las herramientas y estructuras que están relacionadas con las labores productivas y transformadoras del ámbito agrícola y que son reconocibles como tales, elementos que también aportan una información fundamental y que permitirán un futuro trabajo conjunto, que ponga más orden en el entramado de información acerca de los procesos agrícolas.

Habría igualmente que incidir sobre el problema de la calidad y cantidad de los datos que podemos manejar, ya que dependen del nivel de conservación de esas herramientas y esas estructuras, por lo que hay muchos más ejemplos de cierto tipo de elementos que de otros en el registro arqueológico, lo que provocará en el texto que haya tratamientos muy desiguales de los diversos procesos, dependiendo de la cantidad de evidencias con las que contemos en cada caso.

En esta línea, nos gustaría insistir que nuestro objetivo prioritario es llamar la atención sobre los elementos productivos que no están suficientemente documentados en la arqueografía, a pesar de ser más frecuentes en el registro arqueológico de lo que se supone, y de esa forma dar un paso adelante en el conocimiento de las sociedades prerromanas en el sur de la península ibérica, para intentar equilibrar el impresionante desfase entre lo que a este respecto se sabe en el Levante peninsular y la zona de estudio que proponemos en este trabajo.

\section{LA EVIDENCIA ARQUEOLÓGICA}

El registro arqueológico no es muy rico en evidencias relacionadas con el tema que nos ocupa, por la propia naturaleza del mismo así como el papel preponderante de la plantación, recolección, transformación y consumo de nutrientes vegetales; por ese motivo, no contamos con información suficiente para conocer adecuadamente todos los momentos del complejo proceso.

Las tendencias en la investigación han provocado que, a diferencia de los contextos levantinos o septentrionales, el Mediodía peninsular apenas haya sido objeto de estudio en este tipo de temáticas, a pesar de la cantidad de investigación que sobre el mundo ibérico se ha desarrollado.

Pretendemos analizar este registro a lo largo de todos los procesos que tengan que ver con la producción, transformación y consumo de productos cerealísticos, en los que entraría el trabajo agrícola, es decir el cultivo desde la preparación de los campos, pasando por la plantación, la cosecha, el transporte y almacenamiento del producto primario, y finalmente la transformación para consumo final, aunque en cualquiera de estos momentos se puede producir, y de hecho se produce, una retroalimentación a partir de los restos de utensilios y estructuras conservados en el registro arqueológico.

El concepto de producción “....significa la articulación de procesos sucesivos, paralelos y/o acumulativos implicados en la transformación de la materia prima en productos" (Martínez Fernández y Afonso Marrero 1998: 14). Asumiendo esa categoría de análisis, deberemos establecer los protocolos para determinar cada uno de los procesos de producción que intervienen en el paso del cultivo de un cereal hasta su consumo final.

En este caso el procedimiento se inicia siempre integrado en un continuo feedback pues, como comentamos con anterioridad, se retroalimenta de una de las sucesivas fases de producción, siendo intrínsecamente subsecuente a ella. En cualquiera de las fases, incluidas trilla, rastrillado, aventado, criba, tueste, majadura o secado, no todas ellas analizables desde el punto de vista del registro de herramientas o estructural, surgen productos y subproductos que pasan de una a otra de forma casi osmótica (fig. 2).

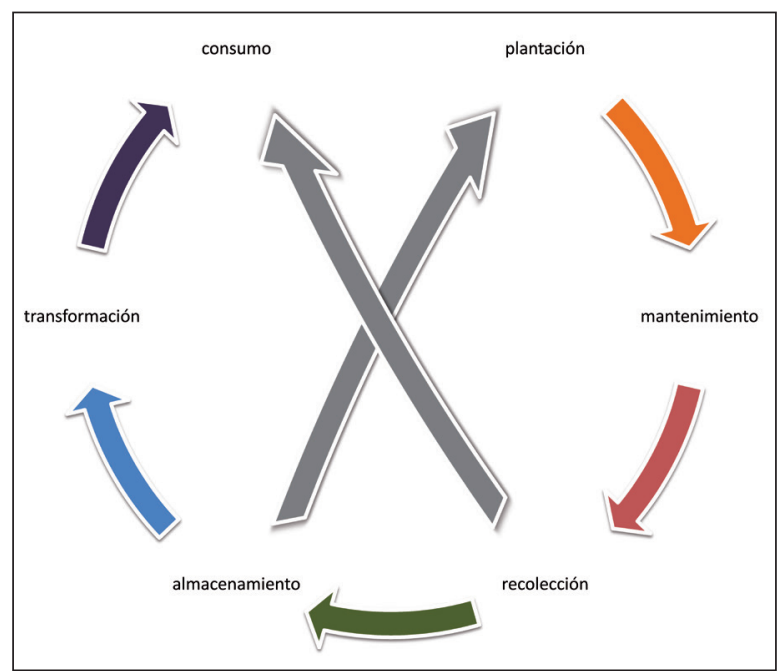

Figura 2. Esquema simplificado del proceso de producción y consumo del cereal en época ibérica. 
La semilla es una materia prima, que no un recurso, desde la perspectiva de que, al menos en el momento que analizamos (Protohistoria peninsular), esta ha surgido de la selección antrópica realizada por el agricultor, por lo que proviene de un cultivo anterior y, en consecuencia, incorpora parte del proceso de producción previo.

Poco sabemos acerca del problema de la preparación de los campos de cultivo; la tala de zonas boscosas y la posterior quema de tocones y otros restos, cuando no su extracción manual, conlleva la utilización de herramientas que, como veremos en diversos momentos del largo proceso analizado, pueden ser multifuncionales, apareciendo y desapareciendo según su funcionalidad en cada uno de los pasos. Hachas para la tala y azadas para la extracción manual de tocones y preparación en general del terreno son instrumentales que vemos utilizados en otros momentos de este proceso productivo (azada), e incluso en otros procesos productivos que nada tengan que ver con la agricultura (hacha). Tras estos pasos se procede al abonado, que se realiza con múltiples sistemas, entre otros la quema de rastrojos, la inclusión de abonos minerales (los estudios de sedimentología y fosfatos podrían informarnos acerca del uso de nitrógeno, potasio o azufre) y de abonos orgánicos, como excrementos (tanto de animales como humanos), humus o cenizas y restos de madera quemada. En este caso la azada sigue jugando un papel importante, o simplemente espera al siguiente paso: el arado.

Una vez que el terreno está convenientemente preparado, se inicia el proceso del arado en el que se prepara el suelo para la recepción de la simiente, como receptáculo donde esta debe germinar. Depende del tipo de cultivo, pero en el ámbito de las gramíneas suele utilizarse casi en exclusiva el arado, sobre el cual hay pocos datos pues la mayor parte de su estructura es de madera, siendo frecuentemente la uña metálica. Si esta fuese igualmente de madera, lo que sucede en algunos casos, no quedaría ningún resto en el registro arqueológico, ya que incluso la ligazón de las distintas partes del arado se realizaría con inserciones de madera (clavos propiamente dichos), no metálicas (como sucede en arquitecturas vernáculas en muchas zonas de estos territorios hasta el siglo pasado).

En el momento de la siembra se produce la reintroducción de la azada y el azadón, herramienta que puede cambiar durante el mantenimiento del cultivo, proceso más o menos complejo dependiendo si el terreno es de regadío o de secano, ya que incluso podría necesitar de la adecuación de elementos de obra constructiva como sistemas de canales de riego, al parecer documentados en ciertos contextos ibéricos tardíos como en Marroquíes
Bajos en Jaén o en Iliberri en Granada, aunque muchos autores dudan de la interpretación dada a esas estructuras.

Finalmente volvemos a cambiar de tipos de herramientas en la siguiente fase, durante la recogida del material, momento en que ya obtenemos otra materia prima, la espiga, de la cual se obtienen dos productos, el grano propiamente dicho y el resto como producto secundario (subproducto, por tanto aún consumible) para consumo animal o para abono, incluso para uso humano como material de construcción (incluido en el adobe), de calentamiento (para fuego) o para muebles (base de lecho).

El proceso de separación del grano de la paja, así como de las malas hierbas se produce posiblemente en espacios preparados para ello, pero que no han sido documentados en el registro arqueológico. A su vez el tratamiento de cada uno de esos subproductos puede ser realizado en zonas diferentes o no, dependiendo de las tradiciones técnicas de ese grupo cultural. El aventado, la trilla, el rastrillado son propios de ciertas zonas posiblemente vecinas al poblado, fuera de los campos de cultivo, pero que no han sido sometidos a ningún estudio. Respecto a las herramientas que se utilizan en estos casos, suelen relacionarse con tridentes que igualmente ayudan al transporte de las balas, como las horcas, que levantan la paja, hacinan las mieses y revuelven la parva. Si parte de la separación se realiza a mano no obtendremos ningún dato, puesto que ese proceso no deja restos visibles directos en el registro arqueológico, aunque sabemos por analogía antropológica que dicha tarea se podría haber realizado con esta técnica.

De este paso obtenemos dos subproductos principales: paja y grano. Mientras que la paja y sobrante en principio no requieren de un mayor tratamiento (en parte serán residuales y en parte subproductos destinados por ejemplo al consumo animal, por lo que pueden ser reprocesados nuevamente), el grano debe ser sometido a nuevos procesos transformativos de producción, que pueden derivar en productos semisólidos, por tanto semielaborados (harina), o líquidos, como la cerveza, en cuyo caso ya tendríamos un producto final. De esta forma, dependiendo de la cadena hacia la que se dirijan sufrirán una transformación u otra. Eso sí, hay que tener en cuenta que una parte de ese grano vuelve a incorporarse al primer proceso de transformación desde la perspectiva que comentamos al principio, que se clasifica, separa y almacena para su posterior utilización en la plantación del año siguiente.

Frecuentemente el grano precisa de un sistema de tostado. El tostado puede tener varias funciones: si vamos a proceder a molienda prepara el grano para desnudarlo mejor y obtener la harina con más facilidad; si 
buscamos desarrollar algún consumo líquido como cerveza, para detener la germinación del grano (aunque puede hacerse cerveza con grano sin tostar); y si finalmente vamos a consumir el grano como tal, entrando en cocina para su directa preparación gastronómica, el tueste reduce el tiempo de cocción e incrementa el sabor de los mismos.

Para la elaboración de la cerveza se necesitarán sistemas de almacenaje para las tres fases necesarias, fermentación, maduración y filtración, tres procesos distintos pero que no requieren una especialización concreta en cuanto al perfil de las vasijas utilizadas (los denominados vasos cerveceros no necesariamente cumplieron esta función y en todo caso, se limitaría a la de servir productos líquidos). El papel de los toneles de madera, si bien empieza a ser conocido en época romana (Marliere 2003) nos resulta del todo desconocido para fases anteriores.

Por el contrario, para la elaboración de la harina recurriremos al proceso de molturación mediante el uso de molinos, generalmente de tipo barquiforme o rotatorio, y estos últimos de mano o de sangre, lo que nos permite inferir procesos y ritmos de producción diferentes.

A su vez la harina, en cuanto producto, pasa a convertirse de nuevo en materia prima en calidad de no consumible directamente, puesto que, a su vez, será manipulada para obtener alimentos en forma de tortas, pan, galletas o gachas, o incluso, pasando a integrarse con otros productos intermedios de carácter alimentario para obtener a su vez otros productos finales más complejos (alimentos donde la harina juegue un papel de componente) tras su cocinado. En este proceso entran de nuevo las vasijas, sea en formato de vasos para preparar los productos (como los morteros), para cocinar (ollas o sartenes) y finalmente hornos para cocer.

Intentaremos colocar cada resto arqueológico en el punto que le corresponda dentro de este amplio y complejo proceso.

\subsection{Instrumental agrícola}

La mayor parte de las herramientas que han llegado hasta nosotros son de hierro, $y$, si generalizamos en el conjunto de la península ibérica, muchas de ellas no tienen una datación contextual anterior a la primera mitad del siglo IV a.C. Eso significa que es precisamente en este momento cuando parece generalizarse la metalurgia del hierro, entre las comunidades indígenas peninsulares en general e ibéricas en particular. Incluso el desarrollo del armamento parece apuntar en esta misma dirección.
Las herramientas de metal forman parte de varios de los procesos intermedios de los anteriormente establecidos. Así en la preparación del terreno, plantación, mantenimiento de esta y recogida, es cuando las herramientas metálicas juegan el papel más claro y más importante. La mayor parte de las hasta ahora recogidas en la documentación arqueográfica parecen relacionarse con estos procesos.

Se han hecho algunos estudios acerca de la funcionalidad de los distintos útiles, como los de Sanahuja (1971) y Barril Vicente (2002), en los que se analiza cada tipo de herramienta agrícola y su posible uso. En este sentido habría que tener en cuenta la multitud de labores que se pueden realizar con un instrumento, $\mathrm{y}$ no encasillar cada tarea con una herramienta determinada, ya que debemos tener en cuenta que para la población campesina, el acceso al utillaje metálico debía ser costoso, y en consecuencia, este sería aprovechado al máximo.

Pasemos a analizar lo que nos ofrece el registro arqueológico. A diferencia de los excelentes ejemplares presentes en los contextos del Levante y Noreste peninsular, la zona meridional apenas ha presentado herramientas relacionadas con el trabajo agrícola.

El primer elemento que podríamos analizar es la reja de arado, aunque no se ha documentado ningún caso en los ámbitos del Mediodía peninsular (Barril Vicente 1999, con abundante bibliografía. En este sentido, en Cancho Roano apareció lo que en un primer momento se catalogó como un puñal y que posteriormente se ha considerado reja de arado. No obstante, hemos preferido no incluir esta pieza debido a que el propio autor que la presenta con esta función desconfía de su contexto (Kurt 2003: 324). Así pues, han sido bien estudiadas las procedentes del yacimiento Mas Castellar de Pontós, en el que han aparecido 5 piezas de los siglos III y II a.C., cuya morfología sería la de láminas triangulares con aletas laterales (Rovira y Teixidor 2002: 363). A pesar de no contar con restos de herramientas de este tipo para el ámbito que estudiamos, sabemos de su existencia, ya que sí han sido encontradas rejas votivas como la de Collado de los Jardines (Santa Elena, Jaén), o representaciones iconográficas como la de las monedas más antiguas de Obulco (Porcuna, Jaén), del siglo III a.C., en las que aparecen rejas de arado junto a otros motivos relacionados con la agricultura como las espigas de trigo. Según Magdalena Barril, las rejas de arado aparecerían en la península ibérica en el siglo IV a.C., siendo las más antiguas documentadas las de La Bastida de les Alcusses (Mogente, Valencia), aunque en otras zonas de Europa se usan desde la Edad del Bronce 

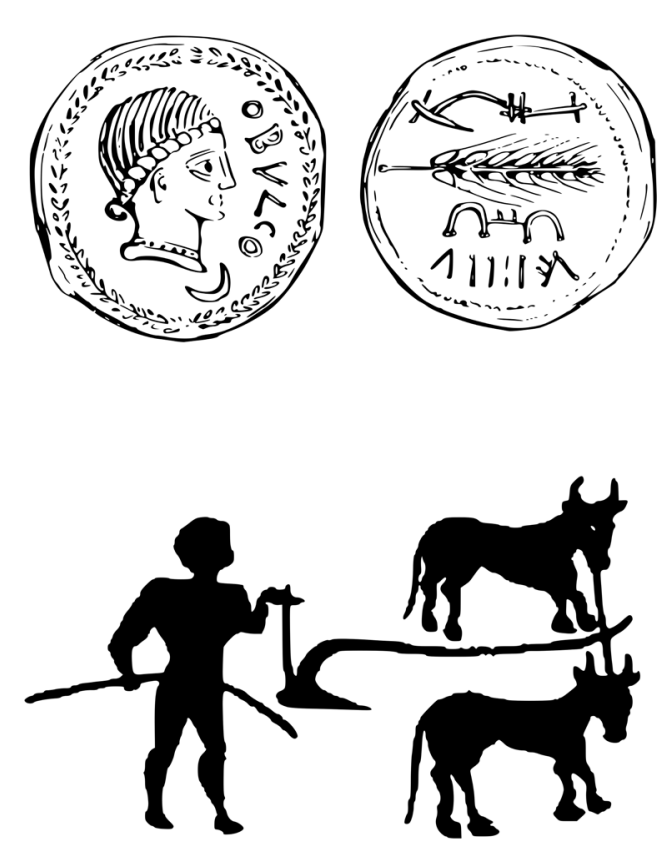

Figura 3. Representaciones iconográficas de instrumental agrícola. Arriba: moneda acuñada en Obulco en la que se pueden apreciar un arado y un yugo junto a la espiga de trigo (López Pérez 2013: 132). Abajo: detalle de la decoración del kalathos del Cabezo de la Guardia (elaboración propia a partir de Atrián y Martínez 1976: fig. 19).

(Barril Vicente 1999). La falta de hallazgos en el Sur puede deberse a que para su fabricación se utilizase exclusivamente material leñoso (fig. 3).

En relación con esta tarea encontramos otros útiles como la aguijada, que también se documenta en la Protohistoria peninsular, concretamente en la Celtiberia, para quitar la tierra que se incrusta en la reja, y que en ocasiones dispone de un látigo en el extremo opuesto para azotar y dirigir a los bueyes (Barril Vicente 1999).

Para conducir los animales de tiro es de suponer que se utilizarían yugos, cuya existencia se constata para este periodo a través de la iconografía, como en el caso del kalathos del arado hallado en el Cabezo de la Guardia (Alcorisa, Teruel), o gracias a representaciones metálicas en miniatura como la del poblado de Covalta (Albaida, Valencia) (Pla 1969: 307) o, como ya hemos visto, la numismática. Sin embargo, por su propia naturaleza perecedera al estar compuestos de madera, no se conservan en el registro arqueológico.

Para cumplir las funciones del arado también se utilizaron otras herramientas de uso manual como la azada, que se documenta en poblados del Noreste peninsular como Mas Castellar (Rovira y Teixidor 2002: 363), Ullastret, Porqueres, Ampurias, o Burriac (Sanahuja 1971: 63).

Tras pasar el arado sería común desterronar la tierra compacta que queda en la superficie, tarea que a lo largo de la historia más reciente se ha hecho gracias a la grada, si bien esta es una herramienta que no hará su aparición en la agricultura peninsular hasta época romana (Mingote Calderón 1996: 94), por lo que cabría pensar que durante la Protohistoria se hiciese manualmente mediante mazos, con la azada o incluso con legones. Son muchos los artilugios que pudieron usarse en esta labor. Columela recomendaba la repetición del arado hasta conseguir que el desterrone posterior no fuese necesario, ya que decía que la tierra bien labrada no necesitaba esta tarea (La Labranza, L. 2, IX), y en al-Ándalus incluso se conoce el uso de rodillos de piedra (Mingote Calderón 1996: 96-97), por lo que la gran variedad de métodos que pudieran realizarse en época ibérica para esta faena se nos escapa en estos momentos. El inconveniente principal es que muchas de las opciones no dejarían constancia en el registro arqueológico, como algunos de los procedimientos comentados con anterioridad. Lo más lógico sería pensar que la realización manual mediante la azada fuese el método más extendido, ya que de las herramientas que constatamos en ese periodo sería la que mejor se adaptase a la función. El hecho de no contar con un útil específicamente dedicado a esta labor hace que se destinen a ello herramientas inicialmente concebidas para otras tareas y que son igualmente válidas para esta, pero que en su caso estarían cumpliendo con una función secundaria. Según Magdalena Barril, la azada sería el útil agrícola más versátil (Barril Vicente 2002: 45), de ahí que pueda usarse en labores muy diversas. Por su parte, el legón también podría servir para dicha tarea, aunque debido a su morfología tiene una menor polivalencia, lo que lo restringe más al ámbito de huertas y a la hora de hacer surcos para el sembrado.

En relación con el abonado se han documentado dos tridentes de época tardía (200-175 a.C.) en Mas Castellar de Pontós, que se han interpretado como elementos usados en la manipulación del estiércol, aunque también pudieron cumplir la función anterior de desterronar tras el arado (Rovira y Teixidor 2002: 363). Para extender el abono según Barril Vicente (2002: 39) el instrumento más extendido serían los rastrillos.

Siguiendo el proceso debemos detenernos en la recolección, lo que nos lleva a los elementos de hoz; en el Sudeste existen algunos datos que podrían informar sobre el hecho de que esta herramienta existe como 
elemento metálico desde el Bronce Final, ya que en Peña Negra se documentó un molde en piedra con nervadura central, que los autores consideran que se trata de la muestra de la fabricación de hoces en bronce, lo cual explicaría la total ausencia de dientes de hoz en estas fases tan recientes de la Prehistoria (González Prats 1990: 86). No obstante, a pesar de ello, los elementos de hoz en piedra tallada siguen siendo mayoritarios, como lo demuestran los ejemplos de Cerro de la Capellanía (Martín Córdoba 1993-1994) y Cerca Niebla (Gran Aymerich et al. 1975), ambos en Málaga en contextos coloniales.

En Cancho Roano, siendo contextos algo posteriores (siglos V y IV a.C., Almagro Gorbea 1991), contamos ya con hoces de hierro plenamente desarrolladas (Kurt 2003: 325-326) (fig. 4). Sin embargo, desconocemos casos intermedios que expliquen cuál ha sido realmente el proceso de cambio que se ha producido en esta herramienta durante el primer milenio a.C. Encontramos también ejemplares de este instrumento en Cáceres el Viejo (Ulbert 1984) (fig. 5) y en Coimbra de Barranco Ancho (Jumilla, Murcia) (Lillo Carpio 1981: 210).

Junto a las hoces se ha planteado el empleo de otros útiles, como las mesories, tradicionales de Asturias, durante la siega ya en época prerromana. Barril Vicente (2002: 41) relaciona esta herramienta, que actúa como una pinza sobre la espiga de cereal, con las "horquillas" a las que hacía referencia Columela cuando hablaba de esta tarea.

Hay, por lo demás, bastantes maneras de segar. Muchos cortan la caña por la mitad con hoces de mango largo, siendo éstas picudas o dentadas; otros muchos cogen solo la espiga con horquillas, algunos con rastrillos, lo cual es facilísimo si el sembrado está claro, pero muy difícil si es denso. (Columela, $\mathrm{La} \mathrm{La}$ branza, L. 2, XXIX).

No obstante es cierto que estas herramientas se relacionan con un tipo de cereal vestido de raquis más quebradizo, propio de la zona asturiana de donde viene el nombre, y posiblemente su radio de acción no debiera ser mayor de la cornisa cantábrica, o que pudieran ser utilizados en casos muy concretos, aunque sería necesario realizar una reflexión más profunda sobre los tipos de cereales que se documentan en cada caso.

Para conocer otras herramientas utilizadas en labores agrícolas en el Sur peninsular, una vez más el registro arqueológico publicado se centra en dos casos de estudio. Uno de ellos trata sobre instrumental en general, que fue realizado por el equipo de Castellones de Céal (Mayoral et al. 1999) acerca del material

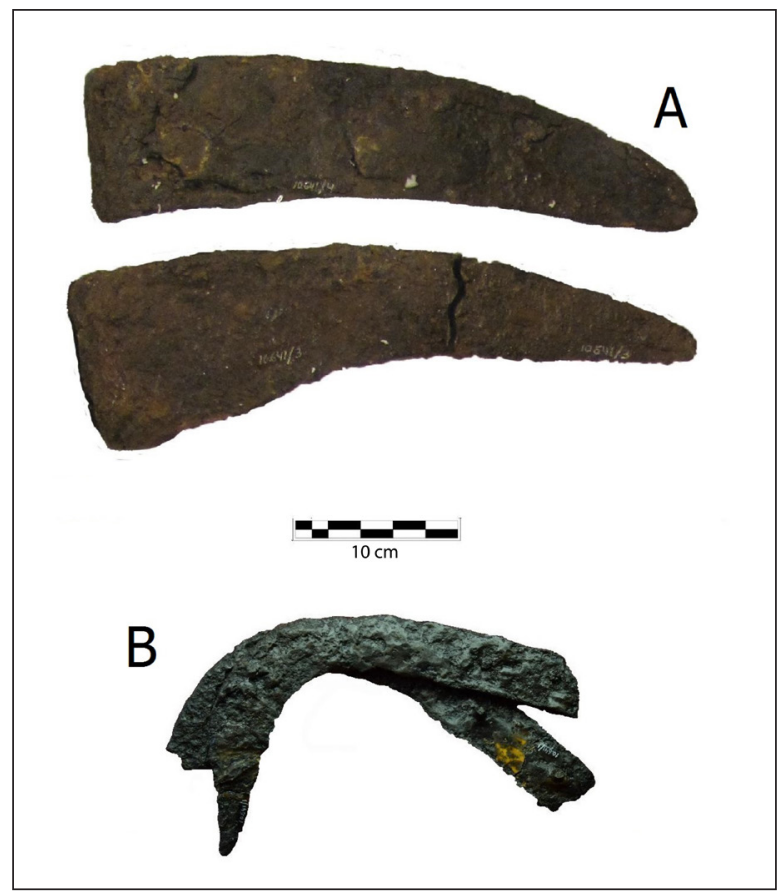

Figura 4. A) Herramienta de siega de Cancho Roano (Almagro Gorbea 1991: fig. 3; Kurt 2003: 326) (Fotografía: Charles Bashore); B) Hoces de Cancho Roano (Kurt 2003: 325) (fotografía: Charles Bashore)

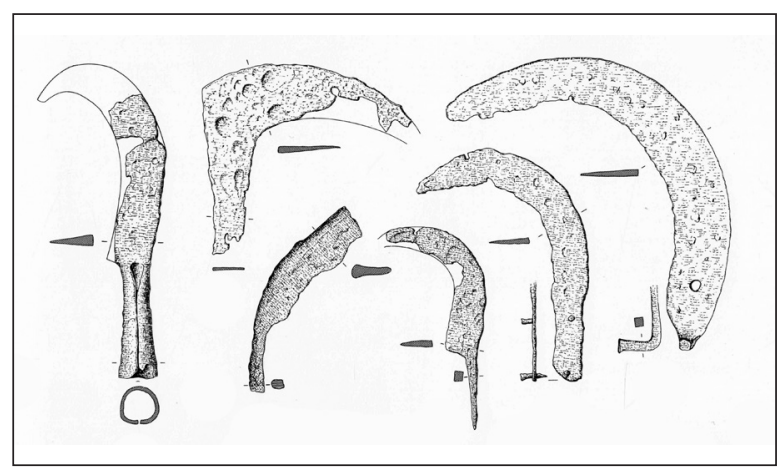

Figura 5. Hoces y podaderas procedentes de Cáceres el Viejo (Ulbert 1984: lám. 27).

aparecido en las viviendas 1 y 2 del poblado; se trata de un conjunto del cual son claramente identificables tres piezas metálicas asociadas a la agricultura: una podadera y un hacha en la vivienda 1 y una aguja en la vivienda 2, todo ello en un ámbito cronológico del siglo II a.C., y que los autores asocian al cultivo del olivo así como a la recolección y tejido de esparto.

Otro conjunto de cronología semejante lo encontramos en el cerro de la Cruz de Almedinilla, en Córdoba 
(Vaquerizo et al. 2001: 222), donde se localizan varios elementos que amplían el repertorio de herramientas relacionadas directa o indirectamente con las labores agrícolas. Tenemos una arrejada de tubo, un posible podón, dos alcotanas mochas y un diente de reja, aunque está muy alterado.

Al igual que en el caso de Castellones de Céal nos encontramos con un conjunto cronológicamente muy tardío, pero en realidad, de acuerdo con lo planteado por algunos autores (Mayoral et al. 1999: 740), el hierro se ha generalizado como material de trabajo desde el siglo V a.C., especialmente desde el punto de vista de la extracción y transformación metalúrgica (Pérez Macías et al. 2003, aunque nos parece más probable la fecha anteriormente propuesta por nosotros mismos de la primera mitad del siglo IV a.C., vs. supra), por lo que no cabe duda de que estas herramientas conocidas en las fases más recientes de la cultura ibérica del Sur peninsular eran ya utilizadas desde el Ibérico Pleno, de modo que no puede argumentarse que la presencia romana $\mathrm{o}$, al menos el contacto en sus primeros momentos con la cultura indígena, tenga nada que ver con el desarrollo de herramientas relacionadas con las actividades agrícolas. Esto no quiere decir que no hubiese una mejora técnica en este sector, pero bien pudo darse por medio de la especialización del uso de los útiles ya existentes, o por nuevos conocimientos relacionados con la forma de hacer las distintas tareas o de abonar la tierra, que no implicasen la utilización de nuevas herramientas.

La separación del grano es un procedimiento complejo de detectar a través de sus utensilios. Si se realizaba majando (con la mano) obviamente no encontraremos restos de herramientas relacionadas, aunque este procedimiento suele utilizarse para pequeñas cantidades (consumo doméstico y puntual); si se hace con mayal tenemos un problema similar, pues este instrumento suele ser entero de madera. Así que el único que podría dejar restos en el registro sería la trilla, siempre que no fuera trilla de pisada (animal o humana), pues nos volvemos a encontrar con la falta de datos sobre el empleo de utensilios (no se utilizan en esos casos). Solamente en el caso del uso de trillo, de tiro animal, podríamos encontrar restos de los materiales utilizados para el corte, bien hojas metálicas o, más probablemente, sílex, que los briqueros (nombre que reciben hoy en la localidad segoviana de Cantalejo los talladores de lascas para las trillas) fabricaban y reponían cada vez que un trillo perdía algunas hojas por rotura, desgaste o pérdida y que, lamentablemente, no han sido objeto de estudio entre las comunidades protohistóricas peninsulares -sobre lo cual advertimos para que sean objeto de mayor atención por parte de los investigadores- salvo algún caso muy colateral, que parece demostrar la existencia de elementos de sílex en la Edad del Hierro (Vallespí 1992-1993), o, más claramente, en época romana (Gutiérrez 2012).

Por otra parte está el problema del trillo de rodillo con paletas, posiblemente de origen cartaginés, conocido como plostellum punicum (Tur y Pons 2005: 108109) y del que no se ha conservado ningún resto a pesar de que las fuentes clásicas parecen mencionar su uso en la península ibérica (Lillo Carpio 1993: 51), donde quizás pudo haberse generalizado a partir de la mitad del siglo III a.C.

Las labores de este tipo se complementan con ciertos procesos como el necesario volteo de la parva para su mejor desbroce, lo que suele hacerse con una tornadera, una horca de dos o tres puntas, normalmente de madera, pero que también podría ser metálica, aunque no se han localizado restos que directamente puedan atribuirse a este tipo de instrumentos, que son del todo iguales a los utilizados en el aventado y otras tareas de separación, que requieren de un desplazamiento de las parvas para mejorar el resultado. Así pues, por el momento tenemos pocas posibilidades de conocer a través de las herramientas conservadas, este proceso de transformación/preparación.

\subsection{Estructuras de almacenamiento}

Dejando de lado el problema del trillado y su evidencia en el registro arqueológico, el almacenamiento es el proceso que sigue a la recogida, selección y preparación del grano, posiblemente con independencia de su destino, sea fabricación de cerveza, harina o conservación para la siguiente plantación.

Al igual que en los apartados anteriores, hay que comenzar haciendo referencia a la gran ventaja que existe en el ámbito del Noreste de la península ibérica con respecto al Sur en cuanto al conocimiento de las estructuras de almacenamiento. En la zona de Cataluña se conocen un buen número de silos, considerándose el sistema preponderante en la conservación del grano en la región, pero en latitudes inferiores no son tan habituales, ya sea por la utilización de otros métodos o por ser menor el recorrido de las investigaciones, que no nos permiten conocerlos (fig. 6).

Uno de los principales problemas que encontramos a la hora de reconocer la existencia de silos es la dificultad que supone conocer la funcionalidad de muchas estructuras negativas que aparecen en diversos 


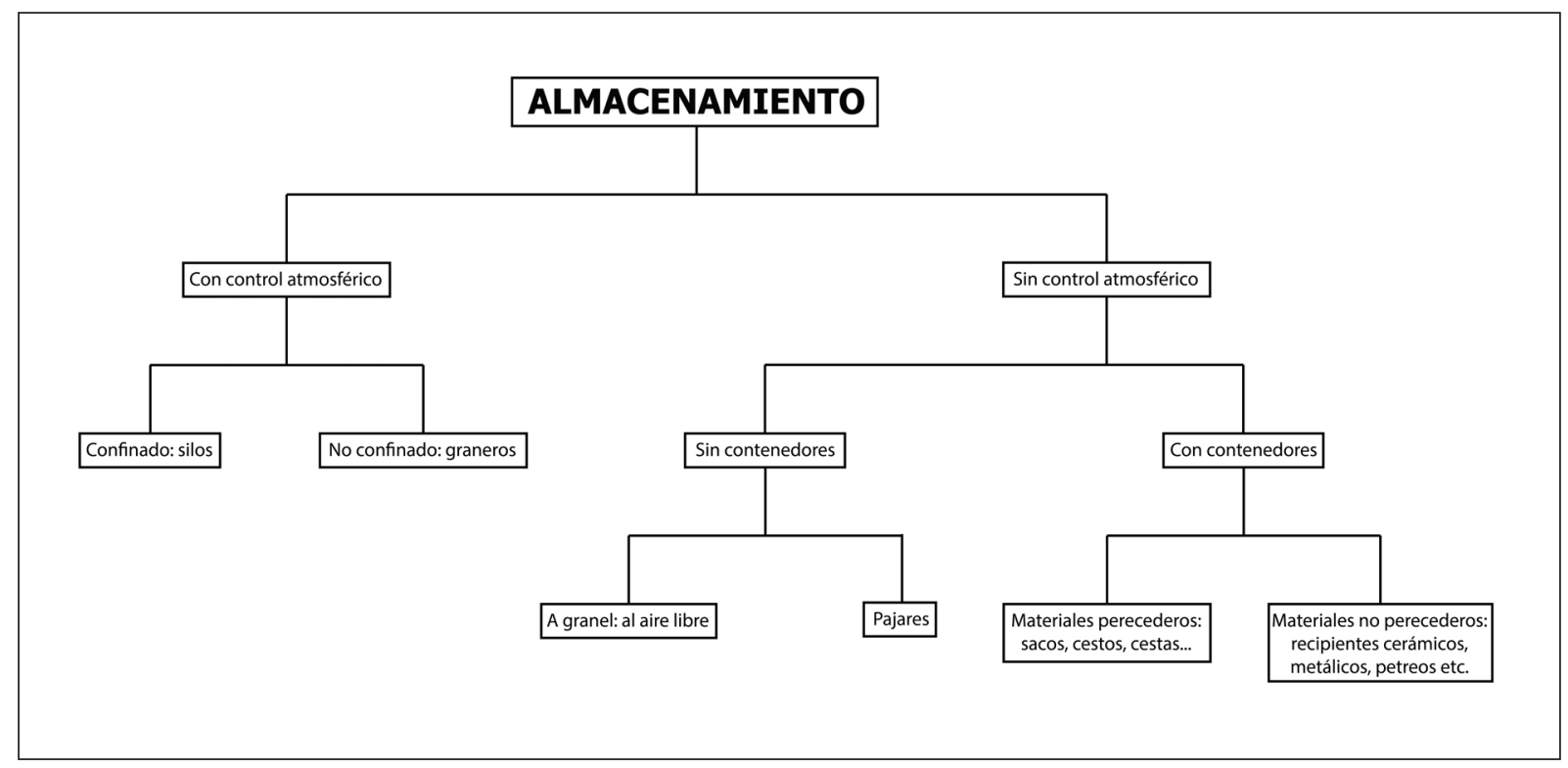

Figura 6. Esquema de los distintos sistemas de almacenamiento susceptibles de haber sido utilizados durante la Prehistoria (elaboración propia a partir de Rovira i Buendía 2007: fig. 28.7).

informes arqueológicos, en los que apenas se proporciona información para poder conocer el alcance de dichas estructuras.

Como ya hemos dicho antes, entre los siglos VI y I a.C. en el área catalana los silos son el sistema de almacenamiento más extendido, desapareciendo paulatinamente en favor del uso de dolia conforme avanza la ocupación romana (Gracia Alonso 2009: 10). Como apuntan Burch y Sagrera (2009: 77) el grano almacenado en silos sería una reserva de cara a un medio y largo plazo, mientras que el cereal necesario para el uso cotidiano se guardaría con otros métodos como los contenedores cerámicos, al tener una menor capacidad al mismo tiempo que una mejor accesibilidad y almacenaje en las unidades domésticas.

A la hora de construir un silo es muy importante el terreno sobre el que se realiza la obra, ya que marcará su eficacia a la hora de conservar el cereal. Los terrenos húmedos e impermeables con pendiente serían los mejores para su emplazamiento según Burch y Sagrera (2009: 75). Estos autores, a pesar de la gran diversidad de tamaños que existe en lo que a silos se refiere, hablan de las ventajas que tienen los de mayor tamaño con respecto a los menores, ya que en los pequeños se perdería una cantidad de grano mayor como consecuencia del contacto con el aire y la humedad (Burch y Sagrera 2009: 76-77). Para evitar este deterioro del cereal los silos se recubrirían de paja después de su construcción y después de introducir el grano, se haría lo mismo con la parte superior antes de sellarlo. Según Varrón, siguiendo este método el trigo se mantenía en buen estado durante cincuenta años (Rerum Rusticarum, L. 1, LVII), afirmación que, aunque desmesurada, muestra la eficacia del sistema (fig. 7).

En relación a posibles silos en el Sur peninsular, contamos con el caso de Villamartín, Cádiz, asociado a fases orientalizantes, con estructuras negativas de 1-2 metros de diámetro y hasta 1'80 de profundidad (Rodríguez González et al. 2009). Ya para época republicana tenemos los ejemplares de Loma de Benagalbón (Efrén et al. 2010: 2598).

Junto a los silos también se documentan almacenes tipo horrea. Según Salido Domínguez (2009: 110) el uso de graneros elevados en el interior de poblados ibéricos se generaliza a finales del siglo $\mathrm{V}$, pero sobre todo desde el III a.C. en adelante. Esto demuestra que no son una implantación romana, sino que simplemente se adecuan a sus necesidades según las diversas circunstancias, puesto que venían construyéndose desde tiempo atrás en el mundo ibérico (Salido 2009: 112).

Tenemos diversos ejemplos de este tipo de estructura en el ámbito meridional de la península ibérica. En primer lugar contamos con dos almacenes elevados de época orientalizante aparecidos en Cerro Manzanillo (Villar de Rena, Badajoz) (Duque et al. 2009: 290). En El Amarejo (Bonete, Albacete) se documentó 


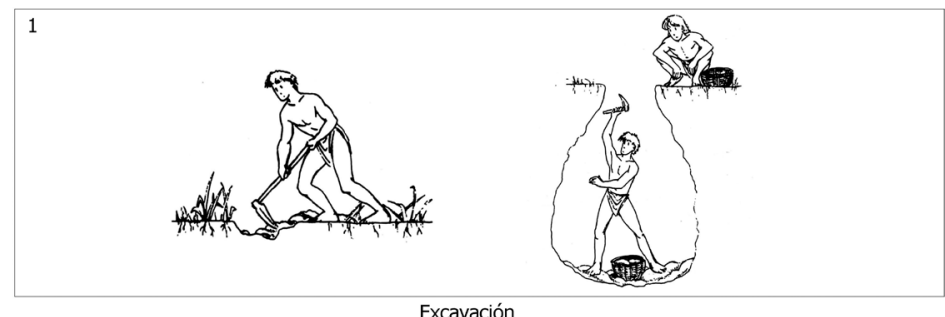

Excavación

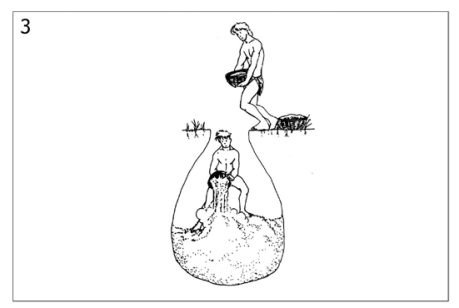

Almacenamiento de grano
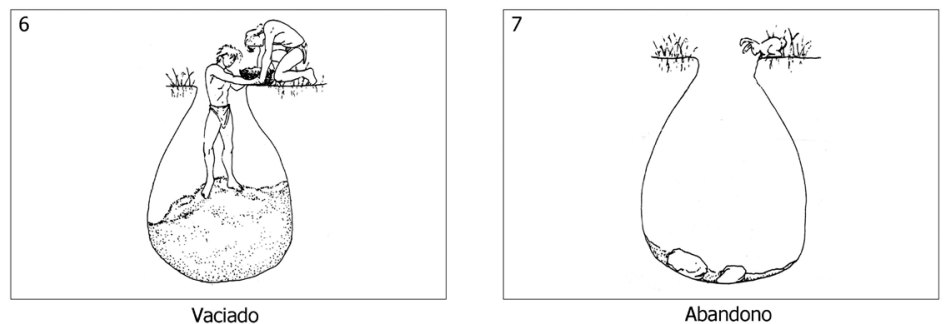

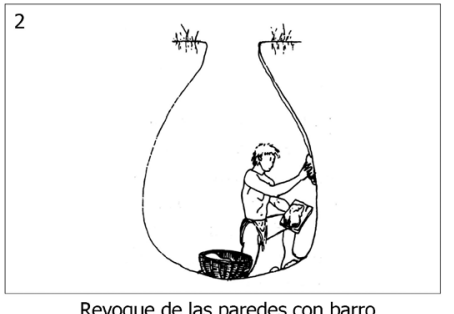

Revoque de las paredes con barro
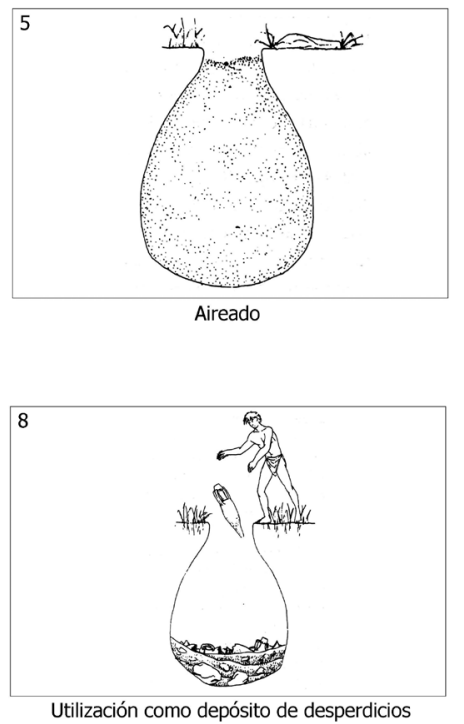

Figura 7. Proceso de construcción y uso de un silo (elaboración propia a partir de Pons 1998: 105).

lo que en primer lugar se interpretó como varios hornos destinados a la fabricación de cerveza, pero que posteriormente se han considerado los restos de un almacén elevado (García Huerta y Morales Hervás 2009: 171). El sector III de Alarcos (Ciudad Real) consta de un edificio con restos de molinos rotatorios, un horno de pan, y abundantes vestigios de contenedores cerámicos y trigo (García Huerta y Morales Hervás 2009: 174-181). Por su parte, el llamado Almacén Q del Cerro de las Cabezas de Valdepeñas (Ciudad Real) se ha interpretado como un lugar de almacenamiento y administración de la producción agrícola del poblado. Consiste en una estructura en forma de hórreo, elevada mediante adobes (García Huerta y Morales Hervás 2009: 183). Visto lo anterior podemos constatar que en la meseta sur el sistema más extendido durante la Protohistoria para almacenar el cereal es el de estas estructuras elevadas.
En otras zonas del Mediodía peninsular encontramos modelos diferentes. En El Palomar (Oliva de Mérida, Badajoz) se encuentra un almacén de época orientalizante en cuyo interior se dispondría el grano directamente en el suelo, formándose cajas de madera que compartimentarían el espacio (Duque et al. 2009: 292). En Puente Tablas (Jaén) el almacenamiento de cereal se realizaba en cada unidad doméstica en pequeñas cantidades destinadas al consumo, reponiéndose cuando se terminaba gracias a otras estructuras de almacenamiento, en este caso comunales, que se situarían cerca de las murallas del poblado (Chapa y Mayoral 2009: 264-265). Esto muestra mucha información sobre la organización social del poblado, ya que nos dice cómo se administra el excedente.

El Cerro de las Cabezas de Valdepeñas aporta buena información sobre este asunto. En primer lugar contamos con una estructura de almacenamiento colectivo, 
Figura 8. BastiónAlmacén del Cerro de las Cabezas (Valdepeñas, Ciudad Real) (Vélez y Pérez 2009: fig. 3).

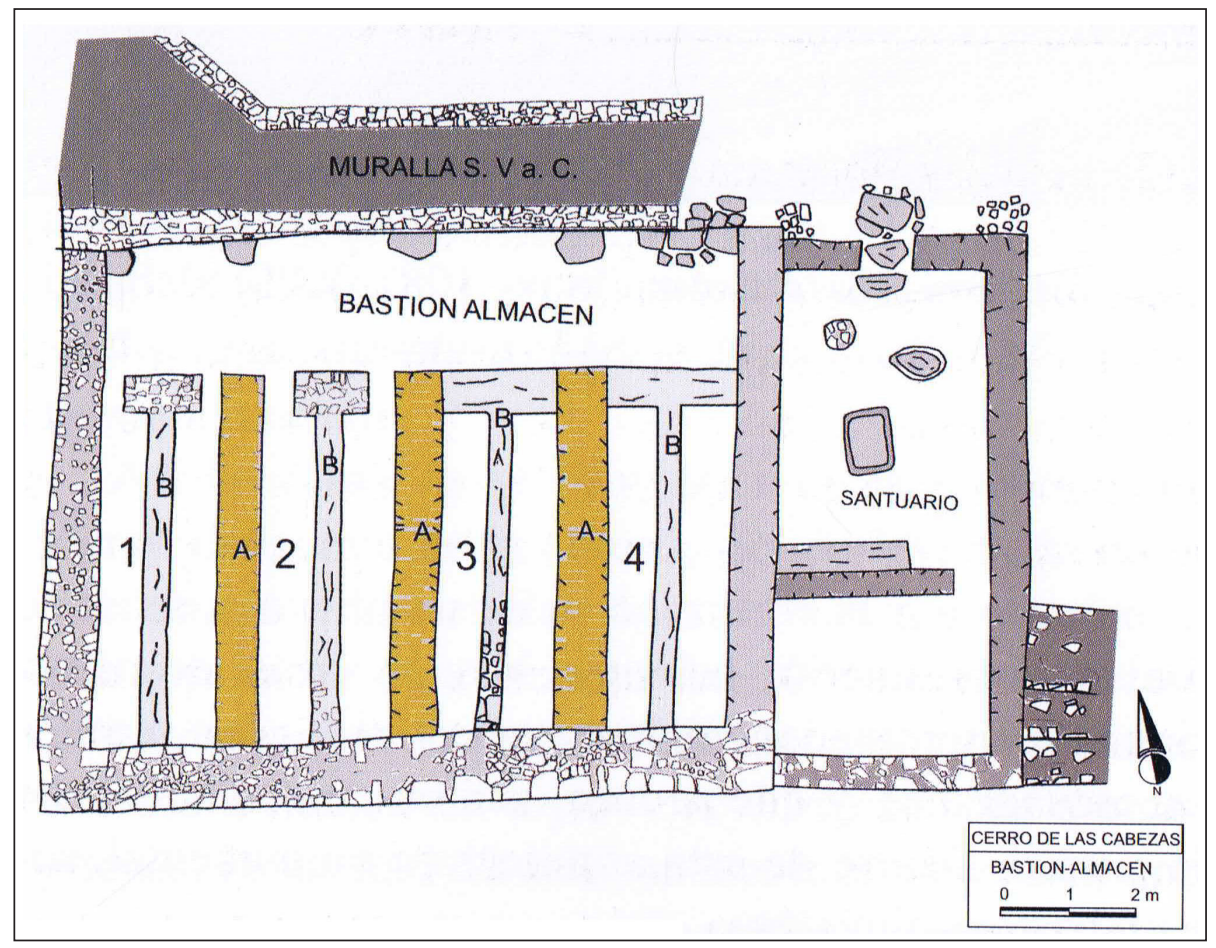

el llamado bastión-almacén (fig. 8); se trata de una construcción defensiva que forma parte de la muralla y que a la vez, en su interior, actúa como un hórreo, guardando el cereal elevado del nivel del suelo (Vélez y Pérez 2009: 253). Por otro lado, la existencia de edificios rectangulares que parecen estar dedicados también al almacenamiento, repartidos dentro del entramado urbano, hace pensar que distintos grupos del poblado, ya sean familiares o de otro tipo, dispondrían de sus propias reservas (Chapa y Mayoral 2009: 266).

Para el Ibérico Tardío es especialmente interesante la información proporcionada por el yacimiento de Castellones del Céal (Hinojares, Jaén). En el complejo I de este asentamiento se han documentado distintas estancias dedicadas al almacenamiento, siendo la que mayor volumen de recipientes cerámicos relacionados con esta función alberga, un patio (Chapa y Mayoral 2009: 267-269).

\subsection{Recipientes de almacenamiento}

Como hemos visto en el apartado anterior, los recipientes cerámicos son muy importantes a la hora de almacenar y conservar los cereales en el Sur peninsular, frente a lo que sucede en el noreste del mundo ibérico, donde los silos son el método más extendido para realizar esta función. De ahí que dediquemos un apartado a los recipientes usados, ya no solo para el almacenaje, sino para cualquier ámbito de la producción cerealista, como el transporte o la transformación del alimento. No vamos a entretenernos mucho en este capítulo, ya que una vez más contamos con escasos datos que nos permitan inferir una relación directa entre cierto tipo de cerámicas y las tareas que hemos mencionado.

En cuanto a las ánforas no parece que sean materiales apropiados para el transporte de cereal, siendo los sacos de cuerda o malla vegetal mucho más funcionales, de modo que aquellas se dedicarían más al transporte de líquidos u otro tipo de alimentos en conserva, a pesar de que en Cerro de la Cruz de Almedinilla muchas de ellas contenían cereales (Quesada et al. 2014), lo que indicaría que se utilizarían como un sistema de almacenaje más que transporte, al menos a media y larga distancia (fig. 9).

En el departamento $\mathrm{n}^{\circ} 5$ de El Amarejo, un ánfora lañada presentaba restos de grano en su interior, unido a un nada desdeñable conjunto de urnas de gran y mediano tamaño apoyadas contra los muros oriental y meridional, utilizadas como contenedores (Alfaro y Broncano 1993: 137).

Los conjuntos de Almedinilla y de Amarejo parecen demostrar que, aunque ciertas formas vasculares son más propicias al almacenamiento de grano, no existe, 


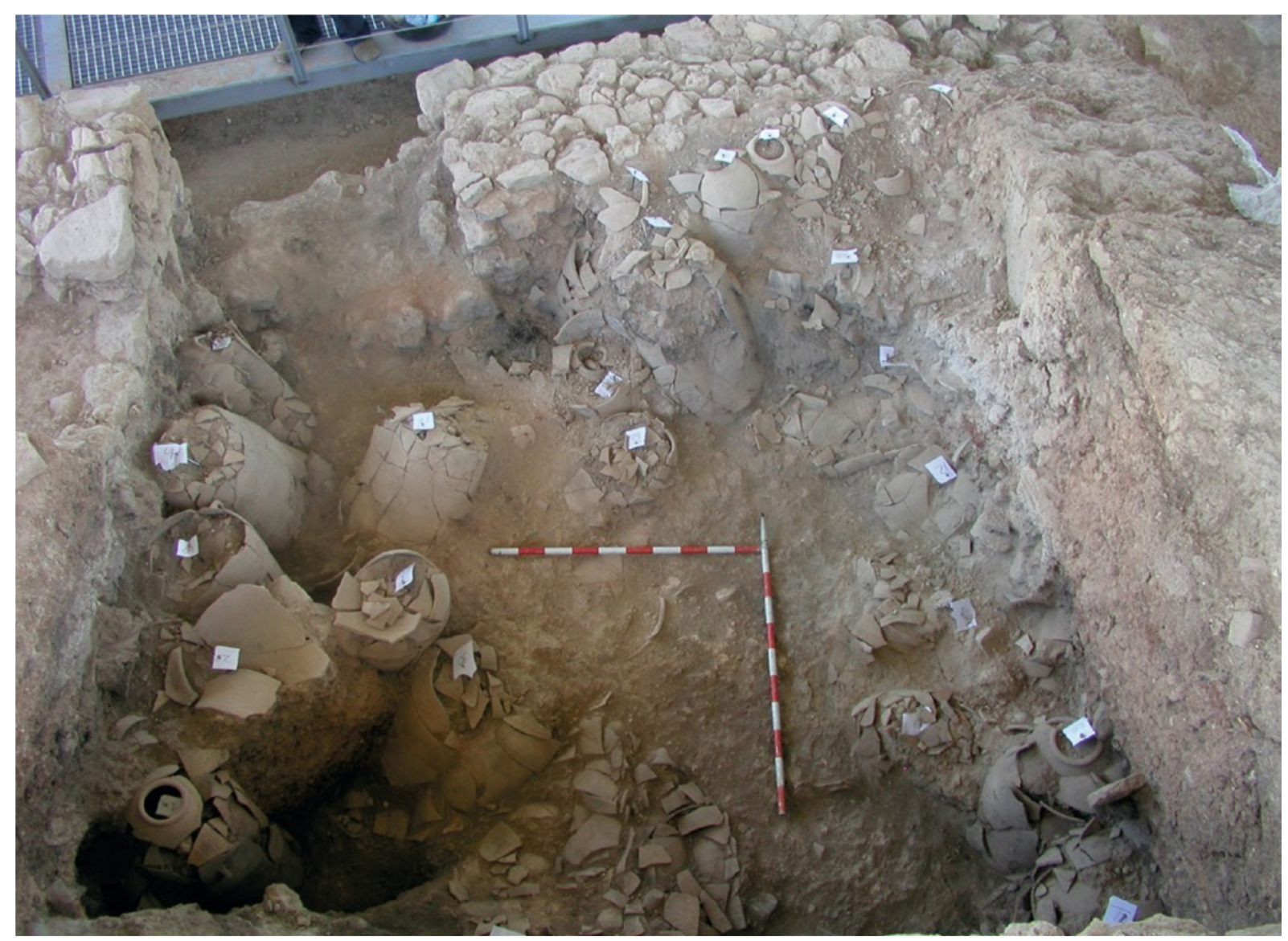

Figura 9. Estancia dedicada al almacenaje de grano en recipientes cerámicos en el Cerro de la Cruz (Almedinilla, Córdoba) (Quesada et al. 2014: fig. 18).

en principio, una especialización en formas únicas destinadas a este fin.

No obstante, en el ámbito del Sureste hay un tipo de tinaja que parece generalizarse a partir del siglo IV a.C., con un importante desarrollo en el III (forma 1a de El Cigarralejo, Cuadrado 1987: fig. 6; Tipo 11.A de Pereira 1988; Grupo I tipo 2.1.2. de Mata y Bonet 1992; clase 52000 de Almedinilla, Vaquerizo Gil et al. 2001). Esta tendencia, y teniendo en cuenta la inexistencia de silos, podría relacionarse con un sistema de almacenaje de carácter doméstico frente al comunitario de los grandes campos de silos del Noreste peninsular; el hecho que se generalice el uso de esta tinaja en torno a los siglos V-IV a.C. podría estar en relación con el incremento de la productividad agrícola que supone el uso sistemático del hierro para la fabricación de herramientas (entre ellas las agrícolas) por parte de las comunidades indígenas. Dichas tinajas son cilíndricas y de boca estrecha, aunque de mayores dimensiones que las ánforas ibéricas, por lo que podrían contener fácilmente un semilíquido, como tradicionalmente se considera al cereal (fig. 10).

Por otra parte, en referencia a la preparación de alimentos, dentro del ámbito colonial existe una interesante propuesta respecto a los cambios culinarios en relación con la forma de los servicios de mesa y cocina; a partir especialmente del contenido (sólido o líquido) y de la forma del fondo de ciertos vasos, se pueden establecer los tipos de cocción que se han desarrollado (Delgado Hervás 2011: 298); de todas formas estas propuestas deberían contrastarse para desarrollarlas como modelos interpretativos.

Resulta interesante indicar la existencia de los platos de hornear, aunque no podemos avanzar muchos datos ante la ausencia de información suficiente al respecto. Solamente podemos indicar que se trata de elementos muebles más propios de ámbitos semitas, ya que, por el momento, no han sido identificados en contextos indígenas (Delgado Hervás 2010: 35). 


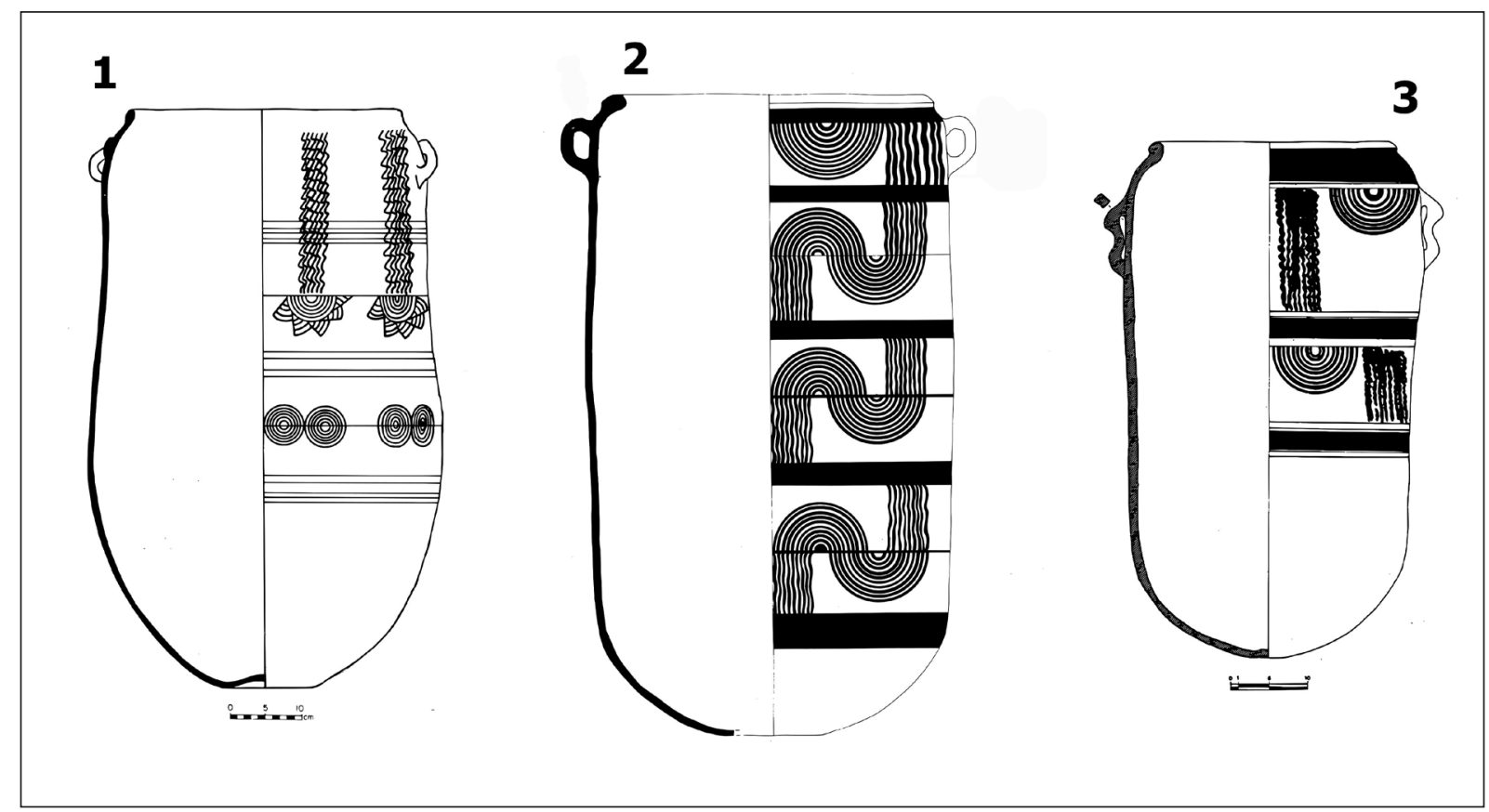

Figura 10. Recipientes cerámicos dedicados al almacenamiento de cereal. 1: Forma 1a de El Cigarralejo (Cuadrado 1987: fig. 82.1); 2: Tipo 11.A de Pereira (1988: fig. 13); 3: Grupo 52000 del Cerro de la Cruz (Almedinilla, Córdoba) (Vaquerizo et al. 2001: fig. 100).

Algunos autores han desarrollado estudios específicamente dedicados a la relación entre los cambios en los servicios de mesa y materiales de almacenaje y transporte y las transformaciones en las habitudes culinarias, si bien nos gustaría destacar el realizado en el entorno del Bajo Guadalquivir, ya que hace interaccionar las cerámicas con datos de paleoambiente y una lectura crítica de las fuentes literarias (García Fernández y García Vargas 2010), señalando las técnicas a utilizar en futuras investigaciones en esta línea.

\subsection{Molinos}

De todos los materiales que componen el proceso de producción de alimentos, los relacionados con la molienda son los más visibles en el registro arqueológico que hemos podido analizar por el momento. No entraremos en detalle sobre la tipología de molinos aparecidos, ya que recientemente se ha publicado un artículo donde se recopilan y analizan todos los hallazgos de este tipo de pieza en el Sur peninsular. Lo que haremos es una breve descripción evolutiva a partir de los datos aportados por este trabajo (Adroher y Molina 2014, con abundante bibliografía).
Los molinos anteriores a la época ibérica son todos de tipo barquiforme. Durante el Bronce Final estos artefactos muestran una reducción en el tamaño de las piezas, lo que los haría más fáciles de transportar y manejables (fig. 11).

Ya en el Ibérico Antiguo encontramos molinos tanto en el interior como en el exterior de las viviendas, lo que implicaría que parte de la actividad se realizaría fuera del ámbito doméstico. En cuanto a su tipología, siguen siendo barquiformes, aunque se apunta la existencia de fragmentos de molino rotatorio en espacios domésticos del Murtal de Alhama, fechados entre los siglos VII-VI a.C. (Lomba y Cano 1999; Adroher y Molina 2014), lo que significaría que estamos ante los restos de molino rotatorio más antiguos de la península ibérica.

El ejemplar de Murtal plantea un serio problema. En primer lugar, cierta imprecisión cronológica; podría estar descontextualizado, presentando un formato intrusivo, en cuyo caso su valor contextual sería despreciable. Sin embargo, de no ser así podría tratarse del ejemplar más antiguo realmente conocido, por lo que se debería considerar que el Sureste peninsular sería el lugar de invención de este tipo de mecánica de molienda, lo que iría en contra de algunas propuestas que señalan 


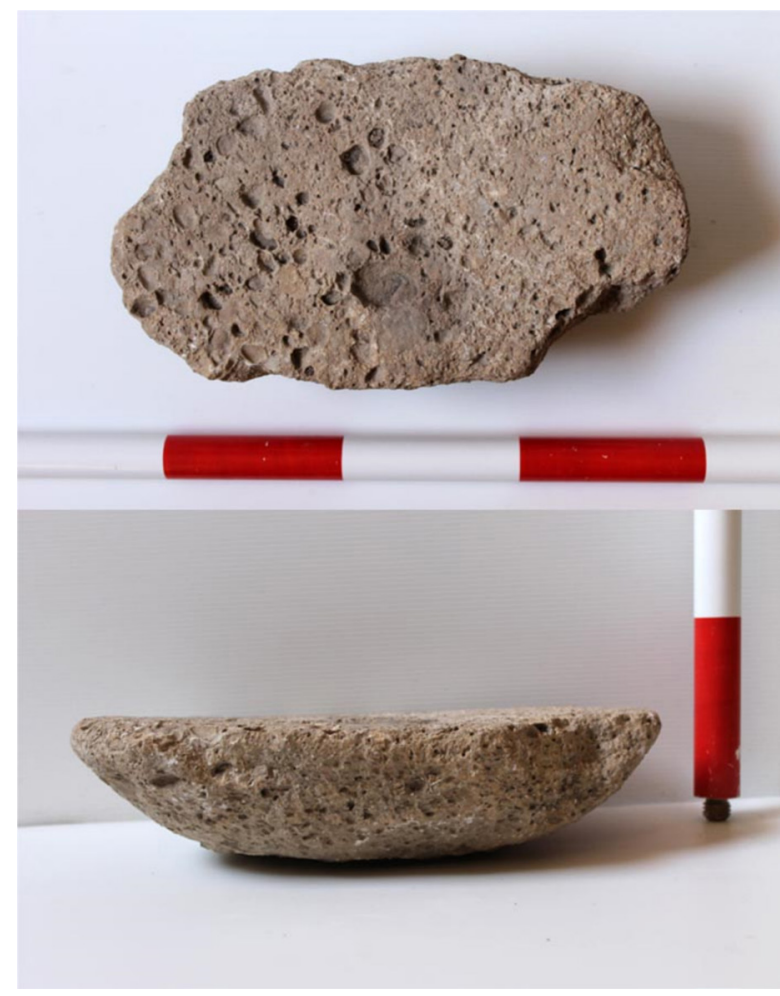

Figura 11. Molino barquiforme del Cerro de los Allozos (Montejícar, Granada) (Adroher y Molina 2014: fig. 7).

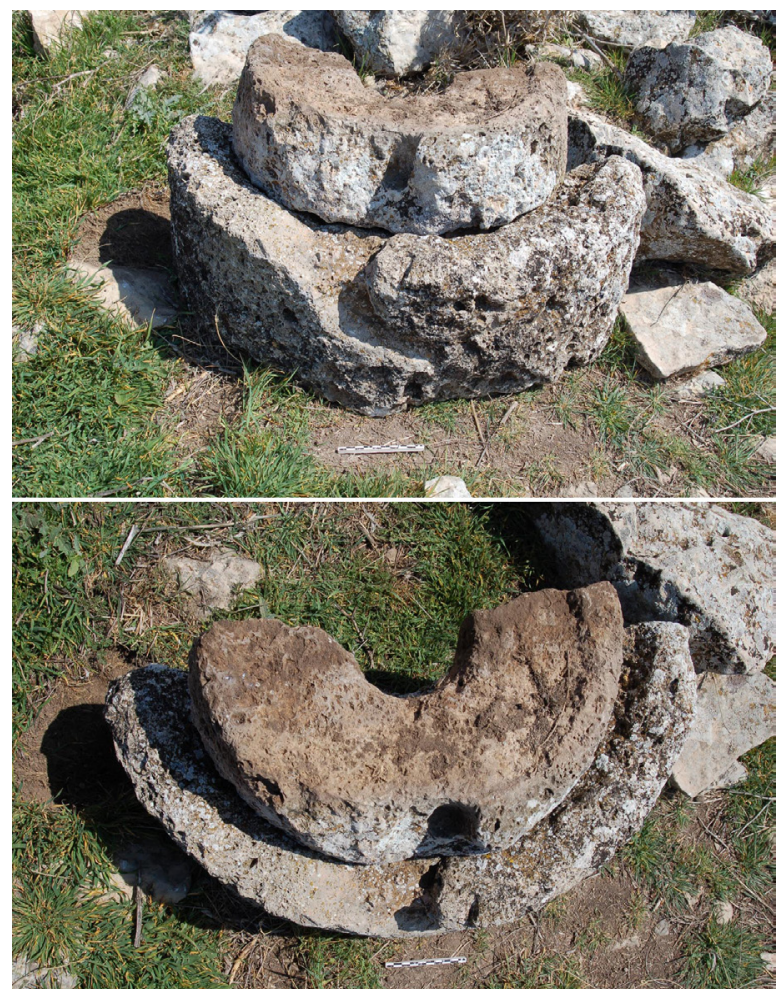

Figura 12. Ruedas de molino del Cerro de la Cruz (Bugéjar, Puebla de Don Fadrique, Granada) (Adroher y Molina 2014: fig. 5). más bien el cuadrante noreste para la invención de dicho artilugio, donde sí existen materiales antiguos con correctas identificaciones contextuales (Alonso y Pérez Jordà 2014); en todo caso el debate está abierto.

Entre los siglos V y III a.C., Ibérico Pleno, se expande el uso del molino rotatorio, aunque no se generaliza. En las zonas más occidentales se han documentado solo molinos barquiformes, pero en el Sureste tenemos piezas rotatorias desde el siglo V a.C., que conviven con las anteriores. Muestra de ello son los hallazgos de la Plaza de Armas de Puente Tablas, en la provincia de Jaén, donde se documenta la presencia de molinos rotarios anteriores al siglo IV junto a barquiformes en contextos que van desde ese siglo hasta el III a.C. También contamos con indicios del comercio de este tipo de material, ya que en la zona de Puebla de Don Fadrique (Granada) existen piezas compuestas por rocas propias del área de Cartagena, con la que la unen buenas vías de comunicación (fig. 12).

Es durante el Ibérico Final cuando parece generalizarse la utilización del molino rotatorio, aunque el uso del barquiforme pervive, sobre todo en asentamientos pequeños y relativamente aislados. En Almedinilla (Córdoba) se ha hecho un gran trabajo que ha permitido establecer una clasificación de molinos, encontrándose algunos in situ, y que pueden ayudar a establecer una tipología si se toman como referencia (Quesada et al. 2014).

En cuanto a los materiales usados en la fabricación de los utensilios de molienda, basándose en el estudio de diez molinos de la zona Guadix-Baza (Adroher y Molina 2014), se aprecia que se abastecían de materias primas próximas para elaborarlos y en los casos en los que se encuentran materiales de otra zona, se trata de lugares conectados con pasos naturales o con una situación que no implica dificultad para establecer relaciones comerciales.

En definitiva podría decirse que el molino barquiforme pierde representación tras la invención del rotatorio, aunque en la Antigüedad Tardía y principios de la Edad Media retoma importancia, ya que el rotatorio desaparece del registro arqueológico durante cuatro siglos (VI-IX).

En cuanto a estudios de funcionalidad, estos son muy escasos. Algunos análisis de la zona de Cataluña 
podrían apuntar hacia la idea de que durante la convivencia del barquiforme y el rotatorio, el primero se usó para productos distintos como ocre rojo, posiblemente como pigmento, bellotas etc., viéndose que, ya en contextos del siglo VI a.C., se detecta su uso para trabajar la arcilla, lo que se podría relacionar con actividades artesanas más que para moler el grano. Para los rotatorios podría hablarse de un comercio a gran distancia y cabe la posibilidad de una zona de producción en Cartagena o Cabo de Gata (Almería), de ahí los materiales propios de esta zona que aparecen en otros lugares, y que se generalice el uso de este molino antes en el Sureste que en otros puntos peninsulares. Finalmente hay que decir que ambos tipos de molinos aparecen tanto en contextos privados como comunitarios.

\subsection{Los hornos de pan}

Dentro de la cadena operativa de la fabricación de alimentos con base cerealística, el horno de cocción supone el último eslabón para la obtención de uno de los productos consumibles.

Una vez más, a este respecto nos encontramos con serios problemas por falta de documentación o, incluso, por una interpretación errónea del registro arqueológico. De hecho solamente conocemos una publicación específica, relacionada con la Oretania (García Huerta et al. 2006).

El más antiguo ejemplo lo tenemos en Córdoba, en Monturque, en el estrato VI, asociado a un molino barquiforme y a restos de cereales, presumiblemente trigo, y se dataría en el Bronce Final, en torno al siglo X a.C. (López Palomo 1999: 154 y 158). En la publicación no encontramos ningún tipo de descripción de la estructura propiamente dicha.

En el yacimiento semita de Chorreras (Málaga), en claro contexto fenicio, se localizó otro horno circular que parece dar servicio a varias unidades domésticas al mismo tiempo (Martín Córdoba et al. 2005).

Más comunes son los ejemplos del siglo VI a.C. y están repartidos por un área mayor. En El Chaparral (Badajoz) tenemos un interesante conjunto, quizás el más complejo hasta ahora conocido, formado por cuatro estructuras circulares de 1,5 metros de diámetro, que pudieran ser interpretadas como hornos de pan, ya que como el propio autor certifica, no pueden ser silos y tampoco hornos metalúrgicos, pues no existen paralelos en este sentido (Sanabria 2008: 46 y 161-142). Sin duda son piroestructuras, en espacios abiertos y en el centro de un conjunto de unidades domésticas que los envuelven, siendo semejantes, desde el punto de vista estructural y siguiendo las planimetrías publicadas, a los otros ejemplares que presentamos aquí.

En Las Calañas de Marmolejo, en Jaén, en el sector 3, con la misma cronología que en el caso anterior, contamos con una estructura circular de apenas un metro de diámetro, que a pesar de la propuesta de horno cerámico por parte de los autores, creemos que más bien pudiera tratarse de un horno de pan que daría servicio a las unidades domésticas que parecen rodearlo en su mayor parte; además, una de las estructuras que se ubican próximas a esta, podría ser considerada como un almacén (Molinos et al. 1994: 22-23).

Siguiendo en la misma centuria, tanto en Chorreras como en Morro de Mezquitilla, ambos asentamientos coloniales fenicios en la costa de Málaga, se detecta la presencia de hornos que se denominan en la bibliografía como de tradición oriental, ubicados dentro de las unidades domésticas, en los patios (como el caso de Chorreras, Martín Córdoba et al. 2005) o incluso al exterior, siendo de uso comunal; además podían existir hornos de pan portátiles, tipo tamuq, habiéndose documentado algún ejemplar en el Cerro del Villar de Málaga (Delgado y Ferrer 2007; Delgado Hervás 2011).

También se ha documentado un horno del siglo VI en el patio de una vivienda en los Almadenes (Hellín, Albacete). Este edificio se ha interpretado como una residencia aristocrática, actuando también de almacén de excedentes (García Huerta y Morales Hervás 2009: 168-169), por lo que el horno pudo ser de uso comunitario a pesar de estar en un contexto aparentemente doméstico, aunque aristocrático; ello demuestra un espacio muy jerarquizado, y sobre todo el control sobre la transformación de cierto tipo de productos.

En el siglo IV contamos con algunos ejemplares más; en el caso de Plaza de Armas de Puente Tablas (Jaén), se ubica junto al palacio del siglo IV a.C., y posiblemente esa relación espacial pudiera permitir considerar que no hay un acceso igualitario al uso de las hornadas, por lo que es posible que desde el siglo VI nos enfrentemos a un control cada vez mayor, por parte de las aristocracias del oppidum, de algunos de los momentos de las cadenas productivas alimentarias.

También al siglo IV pertenece el caso de Alarcos (García Huerta et al. 2006: 158-159); una estructura circular documentada en el sector III, con 1,90 metros de diámetro y una altura conservada de $20-25 \mathrm{~cm}$; los restos de granos y semillas que se asocian a esta estructura ha permitido dar una cronología de ${ }^{14} \mathrm{C}$ cal con el resultado de 370 a.C. Los autores afirman que esta estructura podría haber servido tanto para la elaboración 


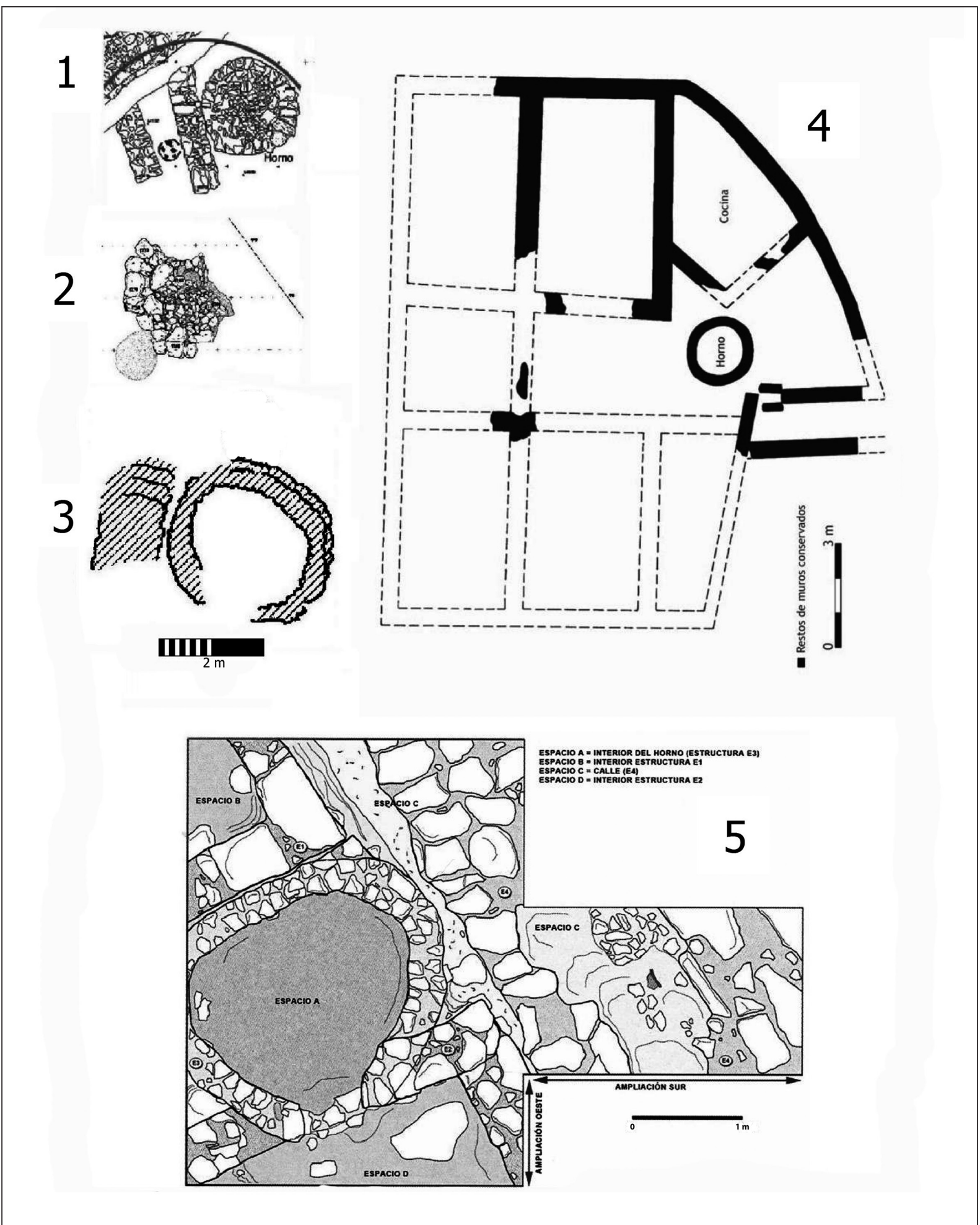

Figura 13. Planimetrías de hornos de pan. 1: Horno de Alarcos (Ciudad Real) (García Huerta et al. 2006: fig. 1); 2: Horno de Calatrava la Vieja (Carrión de Calatrava, Ciudad Real) (García Huerta et al.2006: fig. 2); 3: Horno de Fuente Amarga (Galera, Granada) (Rodríguez Ariza et al. 1999: 289); 4: Horno del yacimiento fenicio de Las Chorreras (Delgado Hervás 2010: fig. 12); 5: Horno de los Turruñuelos (Úbeda, Jaén) (Bellón et al. 2015: fig. 20). 

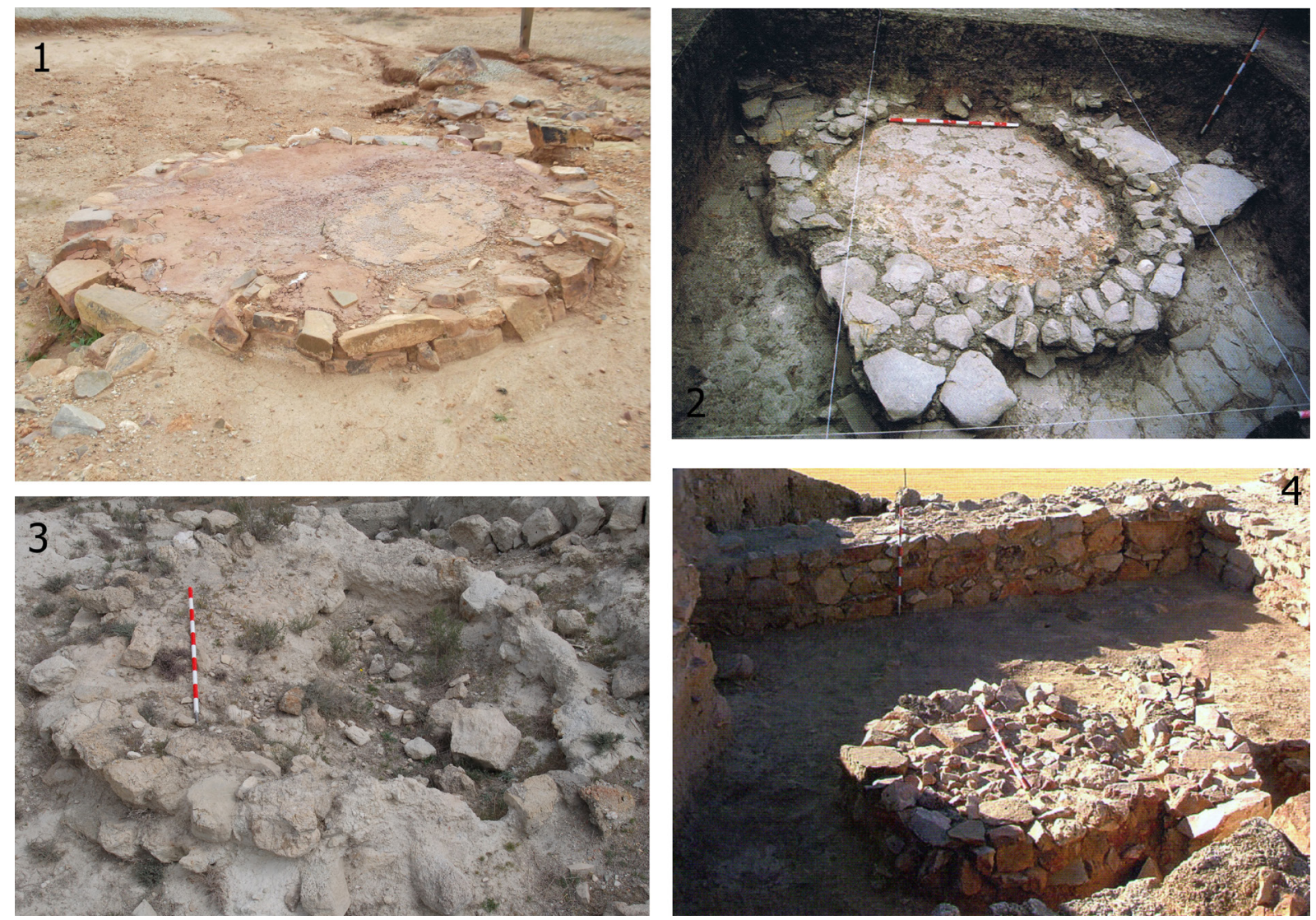

Figura 14. 1: Horno A del Cerro de las Cabezas (Valdepeñas, Ciudad Real) (fotografía: autores); 2: Horno de los Turruñuelos (Úbeda, Jaén) (Bellón et al.2015: fig. 21); 3: Horno de Fuente Amarga (Galera, Granada) (fotografía: autores); 4: Horno de Alarcos (Ciudad Real) (García Huerta y Morales Hervás 2009: fig. 7).

del pan como para el tostado de las semillas para su posterior tratamiento (como la molienda) (fig. 13).

En Los Turruñuelos (Úbeda, Jaén) se ha documentado el pavimento y parte del zócalo de un horno fechado entre los siglos IV y III a.C., en cuyo interior han aparecido restos de semillas de almendro, olivo y trigo (Bellón et al. 2015: 449-451).

Para el siglo III a.C. contamos con tres hornos, uno de ellos en la provincia de Granada, el de Fuente Amarga en Galera (Rodríguez Ariza et al. 1999). Inicialmente fue considerado una estructura relacionada con algún tipo de ritual, pero los estudios más recientes lo interpretan directamente como un horno de pan, al cual se le adosa una pequeña escalera muy simple compuesta de tres peldaños que, de estar vinculada con dicha estructura, facilitaría el acceso a ella (si bien la conexión estructural no queda clara).

También al siglo III a.C. habría que asociar los dos hornos localizados en el cerro de las Cabezas de Valdepeñas, Ciudad Real (García Huerta et al. 2006: 160-163); el primero de ellos, situado extramuros, junto a la zona del almacén de la muralla sur, es circular de 3 metros de diámetro, y se conserva en una altura de $20-30 \mathrm{~cm}$, lo que ha permitido documentar la puerta de acceso orientada hacia el sur. Este hecho ha permitido evidenciar el proceso de construcción del mismo, ya que tras la edificación del muro perimetral en mampostería la parte interior se rellena de una capa de tierra y cascajo, sobre la que se dispone una capa de cantos de río que, a su vez, es subyacente a una tercera compuesta de fragmentos cerámicos dispuestos en plano y sobre esta, una última de arcilla endurecida por efectos de la temperatura. Este método de preparación del horno es generalizable a los sistemas de recuperación y conservación de temperatura en las piroestructuras protohistóricas (Adroher et al. 2001). El segundo horno se ubica en el interior del oppidum ibérico; las medidas y la técnica constructiva son similares a las del anterior, variando solo la altura del muro perimetral que se conserva hasta $40 \mathrm{~cm}$ (fig. 14). 
Sin cronología precisa tenemos, para terminar, el de Calatrava la Vieja (García Huerta et al. 2006: 160). Se trata de dos estructuras circulares muy arrasadas; presentan un diámetro de 2,40 metros y una altura de 15$20 \mathrm{~cm}$ el mejor conservado, que mantenía aún una capa de arcilla en su interior de unos $7 \mathrm{~cm}$ de espesor.

\section{CONCLUSIONES}

Lo primero que queda patente después de analizar los restos arqueológicos relacionados con actividades productivas en el Sur peninsular, es el mayor desarrollo que tiene la investigación de estos aspectos en el área catalana-levantina. La información de que se dispone para cualquier ámbito de la cadena productiva que aquí hemos analizado es muy superior en Cataluña o la Comunidad Valenciana, lo que nos indica que no se les ha otorgado la importancia que merecen a estos restos en las regiones del Sur, situación que nos disponemos
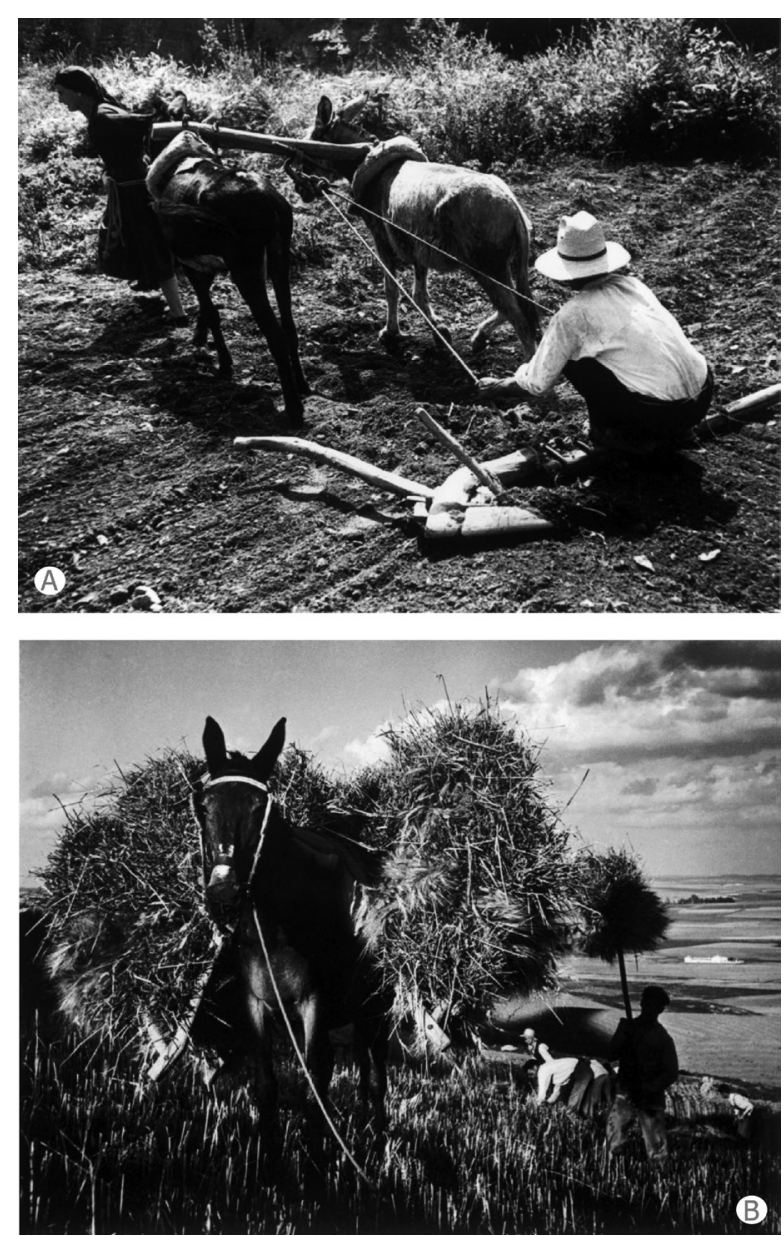

SPAL 26 (2017): 33-57 a denunciar en este trabajo con la intención de que se produzca una inversión en las líneas de investigación.

En lo que al cultivo del cereal se refiere, hay que tener en cuenta la dificultad que supone reconstruir todas las labores que componían este proceso en un periodo tan antiguo. Gracias al instrumental conservado podemos conocer aspectos importantes del trabajo agrícola, pero es de suponer que estamos perdiendo partes de este proceso que no han dejado constancia en el registro arqueológico. De ahí la importancia de los trabajos etnográficos, ya que analizar sociedades anteriores al proceso de mecanización del campo nos puede proporcionar una información valiosa, a pesar del peligro de establecer analogías entre épocas tan distantes, siendo recientes algunos de ellos realizados en países del Magreb (Alonso et al. 2014).

Para la correcta interpretación del uso de los distintos útiles que documentamos también es importante recurrir a la etnografía, ya que como comentamos anteriormente, en muchas ocasiones asociamos cada instrumento a una tarea concreta para la que estaría pensado y nos olvidamos de una realidad mucho más variada, en la que cualquier herramienta es polivalente. De esta forma consideramos un error establecer una sucesión de labores agrícolas basada en unas funciones especializadas de cada instrumento, ya que, aunque sí que existen ciertos útiles que muestran esa especialización, otros son muy versátiles como ya vimos en el caso de la azada (fig. 15).

Como hemos podido comprobar, el instrumental dedicado al cultivo del cereal es prácticamente el mismo que el usado hasta inicios de la segunda mitad del siglo XX en la península ibérica, cuando se produce un profundo cambio en el laboreo agrícola como consecuencia de la mecanización del trabajo en el campo. Es entre los siglos V y IV a.C. cuando se observa una de las mayores innovaciones tecnológicas en el terreno agrario desde el Neolítico, con la introducción del instrumental fabricado en hierro, lo que supone la mejora de rendimientos y la obtención de un mayor excedente para las comunidades campesinas del momento.

Aunque anteriormente ya existían muchas de las herramientas que se usan en este momento, es ahora cuando gracias a la metalurgia del hierro adquieren

Figura 15. A: Un campesino y su esposa arando la tierra antes de la siembra. B: Transporte del cereal recolectado. (Ambas fotografías pertenecen al álbum de Eugene Smith Spanish Village, 1951). 
formas que difieren muy poco de las actuales y aumentan en un grado importante su eficacia.

Pero... ¿qué necesidad existía para adaptarse a este cambio tecnológico? La fabricación de instrumental férreo requiere una especialización técnica y una capacidad tecnológica para fundir el metal muy superior a la que existía con anterioridad, por lo que, si no es necesario aumentar la producción de alimentos, difícilmente una comunidad cambiaría sus utensilios por otros cuya fabricación es mucho más compleja. Quizás en este proceso juegue un papel importante una parte de la población, cada vez más numerosa, dedicada a actividades desligadas de la producción de alimentos y que hace que sus vecinos tengan que aumentar su eficiencia para poder abastecer a la totalidad de la población. En este caso las elites pueden tener un peso esencial, mediante la imposición de una parte de la cosecha que redistribuirían posteriormente, y que obligaría a la población campesina a aumentar su productividad. No debemos olvidar que es debido a la colonización fenicia que la población indígena comience a utilizar el instrumental de hierro, por lo que también pudo deberse a la necesidad de comerciar con los grupos semitas asentados en la costa, especializados en actividades alejadas de la producción de alimentos y que requerirían abastecimiento de productos agrícolas, por lo que proporcionarían el conocimiento técnico necesario a las comunidades autóctonas para incrementar su producción.

En cuanto al almacenamiento del cereal, hemos visto la diversidad de métodos existentes en época protohistórica, y la diferencia existente entre el Sur, donde predomina el grano guardado en recipientes cerámicos, y el Noreste peninsular donde son muy abundantes los silos para esta función. Es difícil precisar si estas diferencias en el método de almacenamiento son solo eso, o si van más allá y nos están mostrando distintos modos de administración de la producción agraria. Sería interesante poder establecer conclusiones acerca de la propiedad y gestión de los recursos almacenados en cada uno de los sistemas, ya que sería fundamental para conocer las relaciones económicas de producción entre los distintos sectores sociales. En este sentido también hay que tener en cuenta que cada método se adapta a una necesidad diferente. El silo está pensado para almacenar el grano a largo plazo, mientras que los recipientes cerámicos permiten sacar producto con frecuencia y además facilitan su movilidad. Es extraño que en el área catalana, más influida por la colonización griega, sea más frecuente el uso del método más inmóvil.
Quizás se deba a que el cereal destinado a la comercialización o al uso cotidiano se conservase en recipientes vegetales (sacos de cuerda), que no encontramos en el registro arqueológico, y los silos se utilizasen para evitar el deterioro del producto almacenado a largo plazo.

Los mismos problemas encontramos con la propiedad de los medios de transformación de los productos agrícolas. Hemos visto cómo el instrumental de molienda aparece tanto en contextos privados como comunes, lo que indica que existiría diversidad en lo que a la gestión se refiere. Es cierto que en los momentos más antiguos, cuando tenemos el predominio del molino barquiforme, lo más frecuente es encontrarlos en contextos domésticos. Pero conforme se va expandiendo el molino rotatorio, especialmente el de tracción animal (conocido como molino de sangre, de mayores dimensiones que el manual) que requiere una mayor inversión para su adquisición y mantenimiento, la transformación del producto se lleva a cabo en lugares compartidos fuera del ámbito doméstico. Lo difícil es llegar a precisar si la gestión de estos medios de producción, que están siendo usados por toda la población, es comunitaria, o si por el contrario es la elite política la que ha acaparado esta parte del proceso y cuenta con el monopolio de la molienda del cereal, pidiendo a cambio algún servicio a la población por su uso, que probablemente sería pagado con una parte del producto llevado hasta el molino.

El caso de los hornos de pan presenta las mismas dificultades que almacenes y molinos. En esta ocasión contamos con trabajos etnográficos como el de Albir (2010) en El Collado, una aldea perteneciente al término municipal de Alpuente, en la Comunidad Valenciana. Con este trabajo, Albir muestra el proceso de elaboración del pan en sociedades campesinas tradicionales a través de una comunidad que sigue usando de forma colectiva el horno de su aldea. Evidentemente asociar los métodos de estas comunidades contemporáneas a las de la Edad del Hierro presenta dificultades, pero sí que puede aportarnos algunas ideas sobre cuál sería la organización y administración de los hornos en caso de que esta fuese comunitaria. Si tomamos la opción contraria y consideramos que estos medios están en manos de las elites del poblado que establecen un monopolio, sería interesante profundizar en el conocimiento de las relaciones que se derivan del uso del resto de la comunidad campesina de los medios de producción puestos a su disposición por las elites políticas (fig. 16). 

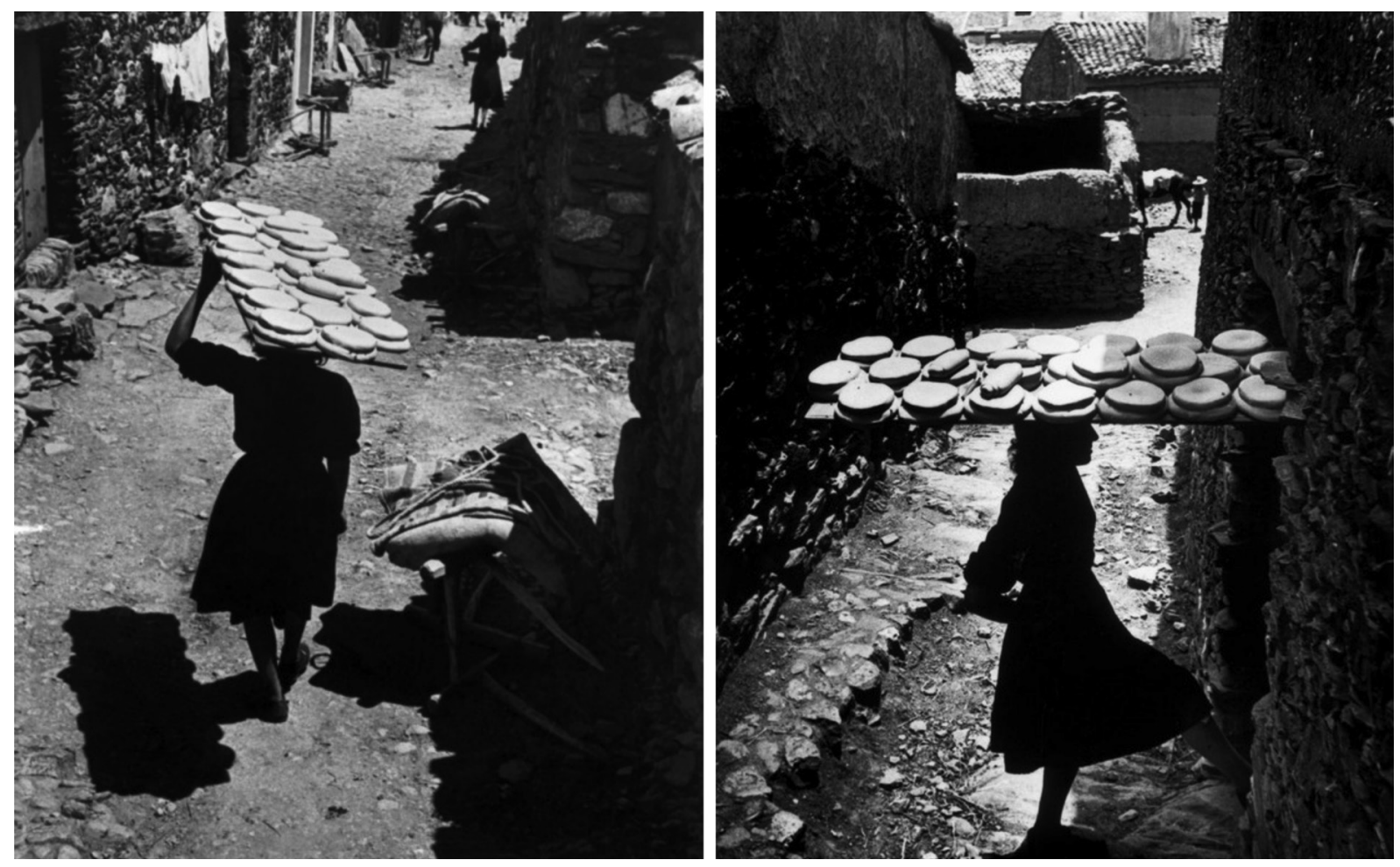

Figura 16. Mujer llevando pan a un horno local a mediados del siglo XX (Fotografías: Eugene Smith Spanish Village, 1951).

\section{BIBLIOGRAFÍA}

Adroher, A. M.; Caballero, A. y López, A. (2001): "Excavación arqueológica de urgencia en la Calle Palacio, s/n (Guadix, Granada)". Anuario Arqueológico de Andalucia, 1997/III: 285-292.

Adroher Auroux, A. M. y Molina Piernas, E. (2014): "La molienda en la protohistoria del mediodía peninsular ibérico". Revista d'Arqueologia de Ponent 24: $215-237$.

Albir, C. (2010): "Etnoarqueología de la elaboración del pan". Saguntum 9 Extra: 151-160.

Alfaro, C. y Broncano, S. (1993): "Estado actual de las excavaciones arqueológicas en el Amarejo", en J. Blánquez; R. Sanz; M.T. Musat (coord.): Arqueología en Albacete. Patrimonio Histórico-Arqueología: 131-146. Madrid, Junta de Comunidades de Castilla-La Mancha.

Almagro Gorbea, M. (1991): "La alimentación en el palacio orientalizante de Cancho Roano". Gerión 3 Extra: 95-114.

Alonso, N. y Pérez Jordà, G. (2014): "Molins rotatius de petit format, de gran format i espais de producción en la cultura ibèrica de l'est peninsular". Revista d'Arqueologia de Ponent 24: 239-255.
Alonso, N.; Cantero, F. J.; Jornett, R.; López, D.; Montes, E.; Prats, G.; Valenzuela, S (2014): "Milling wheat and barley with rotary querns: the Ouarten women (Dahmani, Kef, Tunisia)". AmS-Skrifter 24: 11-30.

Atrián, P. y Martínez, M. (1976): "Excavaciones en el poblado ibérico de Cabezo de la Guardia (Alcorisa, Teruel)". Teruel 55-56: 59-97.

Barril Vicente, M. (1999): "Arados prerromanos de la Península Ibérica: las rejas de arado y su distribución zonal en el interior peninsular", en F. Burillo Mozota (coord.), IV Simposio sobre los Celtiberos. Economía: 89-102. Zaragoza, Institución Fernando el Católico.

Barril Vicente, M. (2002): "Los útiles agrícolas prerromanos: ideas básicas para su identificación, clasificación y adquisición de información". Sautuola 8: 33-56.

Bellón, J.P.; Rueda Galán, C.; Ruiz Rodríguez, A.; Gómez Cabeza, F.; Molinos Molinos, M. (2015): "El oppidum de Los Turruñuelos", en J.P. Bellón, A. Ruiz, M. Molinos, C. Rueda y F. Gómez (eds.), $L a$ Segunda Guerra Púnica en la Península Ibérica. Baecula, arqueología de una batalla: 427-456. Jaén, Universidad de Jaén. 
Blánquez, J.J. (1995): “El poblado ibérico de la Quéjola (San Pedro, Albacete)", en J. Blánquez (ed.), El Mundo ibérico: una nueva imagen en los albores del año 2000: 192-200. Toledo, Junta de Comunidades de Castilla-La Mancha.

Burch, J. y Sagrera, J. (2009): "El almacenamiento de cereales en silos en el Nordeste peninsular. Transformaciones y cambios del Ibérico Pleno al Ibérico Tardío”, en R. García Huerta y D. Rodríguez González (eds.), Sistemas de almacenamiento entre los pueblos prerromanos peninsulares: 73-87. Cuenca, Universidad de Castilla-La Mancha.

Chapa, T. y Mayoral, V. (2009): "Producción y almacenaje de época ibérica en Andalucía Oriental”, en R. García Huerta y D. Rodríguez González (eds.), Sistemas de almacenamiento entre los pueblos prerromanos peninsulares: 257-282. Cuenca, Universidad de Castilla-La Mancha.

Cuadrado, E. (1987): La necrópolis ibérica de El Cigarralejo (Mula, Murcia). Bibliotheca Praehistorica Hispana 22. Madrid, Consejo Superior de Investigaciones Científicas.

Delgado Hervás, A. (2010): "De las cocinas coloniales y otras historias silenciadas: domesticidad, subalternidad e hibridación en las colonias fenicias occidentales", en De la cuina a la taula, IV reunió d'economia en el Ier mil.leni a.C., Saguntum 9 Extra: $27-42$.

Delgado Hervás, A. (2011): “Poder y subalternidad en las comunidades fenicias en la Andalucía Mediterránea", en Memorial Luis Siret, I Congreso de Prehistoria de Andalucía: Tutela del patrimonio Prehistórico: 293-304. Sevilla, Consejería de Cultura de la Junta de Andalucía.

Delgado, A. y Ferrer, M. (2007): “Cultural Contacts in Colonial Settings: The Construction of New Identities in Phoenicians Settlements of the Western Mediterranean". Standford Journal Archaeo$\log y$ 5: 18-42.

Duque, D.; Pérez, G.; Pavón, I. y Rodríguez, A. (2009): "El almacenamiento en la Protohistoria del Guadiana medio: continentes y contenido", en R. García Huerta y D. Rodríguez González (eds.), Sistemas de almacenamiento entre los pueblos prerromanos peninsulares: 283-302. Cuenca, Universidad de Castilla-La Mancha.

Efrén, L.; Salado, J. B. y García Pérez, A. (2010): “Memoria relativa a la prospección arqueológica superficial con carácter puntual del TM de Rincón de la Victoria, Málaga". Anuario Arqueológico de Andalucía 2005/II: 2595-2610.
Falcó Martí, R. (2003): La arqueología de género: espacio de mujeres, mujeres con espacio. Cuadernos de trabajos de investigación 6. Alicante, Universitat d'Alacant.

García Fernández, F. J. y García Vargas, E. (2010): "Entre gaditanización y romanización: repertorios cerámicos, alimentación e integración cultural en Turdetania (siglos III-I a.C.)", en De la cuina a la taula, IV reunió d'economia en el Ier mil.leni a.C., Saguntum 9 Extra: 115-134. Valencia, Universidad de Valencia.

García Huerta, R.; Morales, F. J.; Vélez, J; Soria, L. y Rodríguez, D. (2006): "Hornos de pan en la Oretania septentrional”. Trabajos de Prehistoria 63: 157-166.

García Huerta, R. y Morales Hervás, J. (2009): “Almacenamiento, tratamiento y conservación de alimentos en los pueblos ibéricos de la meseta meridional”, en R. García Huerta y D. Rodríguez González (eds.), Sistemas de almacenamiento entre los pueblos prerromanos peninsulares: 167-207. Cuenca, Universidad de Castilla-La Mancha.

González Prats, A. (1990): Nueva luz sobre la Protohistoria del Sudeste. Alicante, Universidad de Alicante.

Gracia Alonso, F. (2009): "Producción y almacenamiento de excedentes agrícolas en el NE peninsular entre los siglos VII y II a.C. Análisis crítico”, en R. García Huerta y D. Rodríguez González (eds.), Sistemas de almacenamiento entre los pueblos prerromanos peninsulares: 9-71. Cuenca, Universidad de Castilla-La Mancha.

Gran Aymerich, J. M.; Gran Aymerich, E. y Saade, W. (1975): “Cerca Niebla-El Vado 1972". Noticiario Arqueológico Hispánico 3: 141-190.

Gutiérrez, M. (2012): "El uso del sílex en época romana: un indicador arqueológico obviado", en J. Castalheira y C. Gonçalves (eds.), Actas das IV Jornadas de Jovens em Investigação Arqueológica, vol. 2: 227-232. Faro (2011), Faro, Universidade Algarve.

Kurt, G. (2003): "Los hierros de Cancho Roano", en S. Celestino (dir.), Cancho Roano VIII-IX: Los materiales arqueológicos I-II, vol. 1: 293-366. Mérida, Consejería de Cultura de la Junta de Extremadura

Latour, B. (1991): Nous n'avons jamais été modernes. Essai d'anthropologie symétrique. París, La Decourverte.

Lillo Carpio, P. A. (1981): El poblamiento ibérico en Murcia. Murcia, Universidad de Murcia.

Lomba, J. y Cano, M. (1999): “El Murtal. Un sistema fortificado de finales del siglo VII a.C. (Alhama, 
Murcia)", en XXIV Congreso Nacional de Arqueología, vol. 3. Impacto colonial y sureste ibérico: 21 30. Cartagena (1997), Murcia, Instituto del Patrimonio Histórico de la Dirección General de Cultura.

López Palomo, L. A. (1999): El poblamiento protohistórico en el Valle Medio del Genil. Écija, Gráficas Sol.

López Pérez, A. (2013): "Interpretación iconográficonumismática de Obulco y Cástulo”. Trastámara 11 Extra: 129-138.

Mannoni, T. y Giannicheda, E. (2007): Arqueología. Materias, objetos y producciones. Madrid, Ariel.

Marliere, E. (2003): "Tonneaux et amphores à Vindolanda: contribution à la connaissance de l'approvisionnement des troupes stationnées sur la frontière Nord de l'Empire". Vindolanda Excavations 2001-2002, vol. I: 128-179. Bardon Mill, Roman Army Museum Publications.

Martín Córdoba, E. (1993-1994): “Aportación de la documentación arqueológica del Cerro de la Capellanía (Periana, Málaga) a los inicios del primer milenio a.C. en la provincia de Málaga". Mainake 15-16: 5-36.

Martín Córdoba, E.; Ramírez-Sánchez, J. D. y Recio Ruiz, A. (2005): "Nuevo sector urbano fenicio en el yacimiento de las Chorreras (Vélez-Málaga, Málaga)". Ballix. Revista de Cultura de Vélez-Málaga 2: $1-33$.

Martínez Fernández, G. y Afonso Marrero, J.A. (1998): "La producción lítica: un modelo para el análisis histórico de los conjuntos arqueológicos de piedra tallada", en J. Bernabeu, T. Orozco y X. Terradas (eds.), Los recursos abióticos en la Prehistoria. Caracterización, aprovisionamiento e intercambio: 13-28. València, Universitat de València.

Mata, C. y Bonet, H. (1992): "La cerámica ibérica: ensayo de tipología", en Estudios de Arqueología Ibérica y Romana. Homenaje a Enrique Pla Ballester, Servicio de Investigación Prehistórica, Serie de Trabajos Varios del S.I.P. 89: 117-174. Valencia, Museu de Prehistòria.

Mayoral, V.; Chapa, T.; Marcos, F. y Madrigal, A (1999): "Instrumental agrícola del poblado ibérico de Castellones de Céal", en XXIV Congreso Nacional de Arqueología: 735-745. Cartagena (1997), Murcia, Instituto del Patrimonio Histórico de la Dirección General de Cultura.

Mingote Calderón, J. L. (1996): Tecnología agrícola medieval en España. Una relación entre etnología y la arqueología a través de los aperos agrícolas. Madrid, Ministerio de Agricultura, Alimentación y Medio Ambiente.
Molinos, M; Rísquez, C.; Serrano, J. L. y Montilla, S. (1994): Un problema de frontera en la periferia de Tartessos: las Calañas de Marmolejo (Jaén). Jaén, Universidad de Jaén.

Moneo, T. (2003): Religio ibérica. Santuarios, ritos y divinidades (siglos VII-I a.C.), BAH 20. Madrid, Real Academia de la Historia.

Montón Subías, S. (2005): "Las prácticas de alimentación: cocina y arqueología”, en M. Sánchez (ed.), Arqueología y Género, Monografías Arte y Arqueología: 159-176. Granada, Universidad de Granada.

Pereira, J. (1988): "La cerámica ibérica de la cuenca del Guadalquivir. I. Propuesta de clasificación". Trabajos de Prehistoria 45: 143-173.

Pérez Macías, J. A.; Guerrero Chamero, O. y Serrano Pichardo, L. (2003): "El Cerquillo (Cerro de Andévalo, Huelva) y la producción metalúrgica prerromana en el suroeste ibérico". Huelva en su historia 20: 9-28.

Pla, E. (1969): "El instrumental metálico de los obreros ibéricos", en X Congreso Nacional de Arqueología: 306-337. Zaragoza, Universidad de Zaragoza.

Pons Brun, E. (1998): "Los silos en época ibérica", en VV.AA., Los Iberos. Príncipes de occidente: 104107. Barcelona, Association Française d'Action Artistique, Ministerio de Educación y Cultura, Fundació "la Caixa", KABD Bonn.

Quesada, F.; Kavanagh de Prado, E. y Lanz Domínguez, M. (2014): "Los molinos del yacimiento del Cerro de la Cruz (Almedinilla, Córdoba): clasificación y análisis de los ejemplares de época ibérica y emiral". Spal 23: 83-118.

Rodríguez Ariza, M . O.; Fresneda Padilla, E.; Peña Rodríguez, J. M. y López López, M. (1999): “Los niveles ibéricos de Fuente Amarga (Galera, Granada)", en XXIV Congreso Nacional de Arqueología: 283-292. Murcia, Instituto del Patrimonio Histórico de la Dirección General de Cultura.

Rodríguez González, G.; Pineda, S. y Navarro, M. (2009): "La intervención arqueológica de urgencia en la unidad de ejecución 1, fase II A-B. Torrevieja Alta, Villamartín, Cádiz". Anuario Arqueológico de Andalucía 2004, I: 261-269.

Rovira, M.C. y Teixidor, E. (2002): "Els objectes metàl.lics i el treball del metal", en E. Pons (dir.), Mas Castellar de Pontós (Alt Empordà). Un complex arqueolòic ibéric (excavacions 1990-1998): 333367. Girona, Generalitat de Catalunya, Departament de Cultura.

Rovira i Buendía, N. (2007): Agricultura y gestión de los recursos vegetales en el sureste de la Península 
Ibérica durante la prehistoria reciente. Tesis doctoral, Universitat Pompeu Fabra, Barcelona. http:// www.tdx.cat/handle/10803/7468 (última consulta 28/10/16).

Salido Domínguez, J. (2009): “Transformación y evolución de los sistemas de almacenamiento y conservación de excedentes agrícolas desde la Edad del Hierro a la época romana”, en R. García Huerta y D. Rodríguez González (eds.), Sistemas de almacenamiento entre los pueblos prerromanos peninsulares: 103116. Cuenca, Universidad de Castilla-La Mancha.

Sanabria, D. (2008): Paisajes rurales protohistóricos en el Guadiana Medio: El Chaparral (Aljucén, Badajoz). Memorias de Arqueología Extremeña 10. Mérida, Editora Regional de Extremadura.

Sanahuja, M. E. (1971): "Instrumental de hierro agrícola e industrial de la época ibero-romana en Cataluña". Pyrenae 7: 61-110.

Tur, J.A. y Pons, A. (2005): "La alimentación en el mundo púnico", en J. Salas-Salvadó, P. García-Lorda y J.M. Sánchez-Ripollès (eds.), La alimentación y la nutrición a través de la Historia: 87-111. Barcelona, Editorial Glosa.

Ulbert, G. (1984): Cáceres el Viejo. Ein spätrepublikanisches Legionslager in Spanisch-Extremadura. Mainz, Philipp von Zabern.

Vallespí, E. (1992-1993): "Piezas líticas y talleres domésticos en los poblados hallstáticos del Bajo Aragón”. Bajo Aragón, Prehistoria 9-10: 71-82.

Vaquerizo Gil, D.; Quesada Sanz, F. y Murillo Redondo, J. F. (2001): Protohistoria y romanización en la subbética cordobesa. Una aproximación al desarrollo de la cultura ibérica en el sur de la actual provincia de Córdoba. Arqueología Monografías 11. Sevilla, Junta de Andalucía.

Vélez, J. y Avilés, J. J. (2009): "El oppidum del Cerro de las Cabezas (Valdepeñas, Ciudad Real). El bastión almacén de la muralla sur", en R. García Huerta y D. Rodríguez González (eds.), Sistemas de almacenamiento entre los pueblos prerromanos peninsulares: 241-256. Cuenca, Universidad de Castilla-La Mancha. 